\title{
Infrared photometry and evolution of mass-losing AGB stars
}

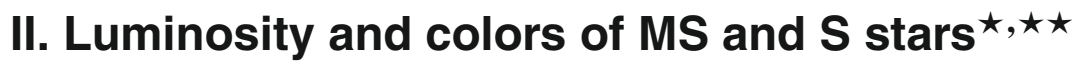

\author{
R. Guandalini and M. Busso \\ Department of Physics, University of Perugia, and INFN, Sezione di Perugia, via A. Pascoli 1, 06123 Perugia, Italy \\ e-mail: [guandalini; busso]@fisica.unipg.it
}

Received 8 April 2008 / Accepted 24 June 2008

ABSTRACT

\begin{abstract}
Context. Asymptotic giant branch (AGB) phases mark the end of the evolution for low- and intermediate-mass stars. Our understanding of the mechanisms through which they eject the envelope and our assessment of their contribution to the mass return to the interstellar medium and to the chemical evolution of Galaxies are hampered by poor knowledge of their luminosities and mass loss rates, both for C-rich and for O-rich sources.

Aims. We plan to establish criteria permitting a more quantitative determination of luminosities (and subsequently of mass loss rates) for the various types of AGB stars on the basis of infrared fluxes. In this paper, in particular, we concentrate on O-rich and $s$-elementrich MS, S stars and include a small sample of SC stars.

Methods. We reanalyze the absolute bolometric magnitudes and colors of MS, S, SC stars on the basis of a sample of intrinsic (single) and extrinsic (binary) long period variables. We derive bolometric corrections as a function of near- and mid-infrared colors, adopting as references a group of stars for which the spectral energy distribution could be reconstructed in detail over a large wavelength range. We determine the absolute HR diagrams, and compare luminosities and colors of S-type giants with those, previously derived, of C-rich AGB stars. Luminosity estimates are also verified on the basis of existing period-luminosity relations valid for O-rich Miras. Results. S star bolometric luminosities are almost indistinguishable from those of C-rich AGB stars. On the contrary, their circumstellar envelopes are thinner and less opaque. Despite this last property the IR wavelengths remain dominant, with the bluest stars having their maximum emission in the $H$ or $K$ (short) bands. Near-to-mid infrared color differences are in any case smaller than for $\mathrm{C}$ stars. Based on period-luminosity relations for O-rich Miras and on Magnitude-color relations for the same variables we show how approximate distances (hence intrinsic parameters) for sources of so far unknown parallax can be inferred. We argue that most of the sources have a rather small mass $\left(<2 M_{\odot}\right)$; dredge-up might then be not effective enough to let the $\mathrm{C} / \mathrm{O}$ ratio exceed unity.
\end{abstract}

Key words. stars: fundamental parameters - stars: AGB and post-AGB - stars: evolution - infrared: stars - stars: variables: general

\section{Introduction}

The asymptotic giant branch phases (hereafter AGB) represent the second ascent along the red giant branch, occurring after the exhaustion of core He burning for all stars between $\sim 0.8$ and $8.0 M_{\odot}$. In these evolutionary stages, stars are powered by two nuclear shells, burning $\mathrm{H}$ and $\mathrm{He}$ alternatively. In particular, in the final 1-2 Myr of the AGB, the He-burning shell remains mainly quiescent, if not for recurrent explosive ignitions during which a lot of $\mathrm{C}$ (from 20 to $25 \%$ by mass) is produced and spread over the whole He-rich layer, in short phases of convective mixing (the so-called thermal pulses). Convective penetration of the envelope follows, in repeated episodes collectively called "the third dredge-up", and carries the new carbon to the surface, together with other nucleosynthesis products, in particular $s$-elements generated by efficient neutron captures (Busso et al. 1999).

Due to the above phenomena, the atmospheres of AGB stars are characterized by an increasing enrichment of ${ }^{12} \mathrm{C}$ (up to $\mathrm{C} / \mathrm{O}>1$ by number, in which case we speak of $\mathrm{C}$ stars) and of $s$-process nuclei, in particular revealing the recent nucleosynthesis through the short-lived ${ }^{99} \mathrm{Tc}$ (Merrill 1952).

\footnotetext{
* Tables 1-10 are only available in electronic form at http://www . aanda.org

$\star \star$ Tables 11 and 12 are only available in electronic form at the CDS via anonymous ftp to cdsarc.u-strasbg.fr (130.79.128.5) or via http://cdsweb.u-strasbg.fr/cgi-bin/qcat?J/A+A/488/675
}

Sometimes Tc itself offers actually the only real evidence of ongoing $s$-processing in stars that do not show other remarkable chemical anomalies (Uttenthaler et al. 2007). When instead the third dredge-up process is efficient enough, changes in the photospheric abundances of other $s$-elements begin to occur, first of all for $\mathrm{Zr}$, which has various isotopes on the main s-process path. In such cases the appearance of $\mathrm{ZrO}$ bands in the spectra (at wavelengths 464.1, 462.0, 530.4, 537.9, and $555.1 \mathrm{~nm}$ ) tells us that the star, although still richer in $\mathrm{O}$ than in $\mathrm{C}$, is mixing to the surface the products of shell-He burning. The cool giants presenting these signatures are called MS and S stars (the second group showing more prominent features). They also have a $\mathrm{C} / \mathrm{O}$ abundance ratio by number higher than in the Sun, but lower than unity. There is still some confusion about the exact values of the $\mathrm{C} / \mathrm{O}$ ratios in MS and $\mathrm{S}$ stars, which is mainly induced by the remaining uncertainty in the calibrating solar oxygen abundance. If one excludes for this calibration the recent, still debated suggestion (Allende Prieto et al. 2002) and adopts instead the previous more traditional reference (Anders \& Grevesse 1989), then MS stars are found typically at $\mathrm{C} / \mathrm{O}=0.5$ to 0.7 , and S stars are found above this range and up to more than 0.95. Around the border between $\mathrm{O}$-rich and $\mathrm{C}$-rich giants, the so-called SC stars represent a rare, but important, transition group.

In some cases, red giants showing $s$-element enhancement are Tc-poor. This is a clear indication that a sufficiently long time interval has passed since the production of neutron capture nuclei, so that Tc has decayed. In general, this is the case when 
the nucleosynthesis phenomena occurred not in the same star we see today, but in a more massive companion, which is now evolved to the white dwarf stage, and whose mass loss enriched in the past the photosphere of the observed object (Busso et al. 2001). In these cases we speak more properly of an extrinsic AGB star (Smith \& Lambert 1990).

AGB stars lose mass very effectively, and their winds replenish the interstellar medium (ISM) guaranteeing up to $70 \%$ of the mass return from stars (Sedlmayr 1994). Before being dispersed over the Galaxy, the material thus lost forms cool envelopes (Winters et al. 2003) where dust grains condense (Carciofi et al. 2004). These solid particles carry the elemental and isotopic composition generated by AGB nucleosynthesis; they have been found in ancient meteorites offering the possibility of high precision isotopic abundance measurements on matter coming from circumstellar environments (e.g. Zinner 2000).

The cool AGB photospheres radiate most of their flux at red-infrared wavelengths. The infrared component of the spectral energy distribution (SED) grows in importance (and in average wavelength) as far as the evolution proceeds, because of the increased extinction of the photospheric flux operated by dust, which then re-radiates at long wavelengths (see e.g. Habing 1996). This correlation between extinction and evolutionary stage is however confused by the stars switching from Semiregular to Mira-type surface variability, which fact modulates the mass loss efficiency and hence the extinction properties. Due to these complicacies, large surveys of infrared (IR) observations play a fundamental role in studying luminosities and mass loss rates of AGB stars and in disentangling the variability and evolution effects (see e.g. Wood \& Cohen 2001; Le Bertre et al. 2001, 2003; Groenewegen et al. 2002; Cioni et al. 2003; Omont et al. 2003; Olofsson et al. 2003).

Longstanding efforts have been devoted to describe the mass loss mechanisms, either with phenomenological models or with sophisticated hydrodynamical approaches (Salpeter 1974; Knapp \& Morris 1985; Winters et al. 2003; Wachter et al. 2002; Sandin \& Höfner 2003a,b). Despite this, our quantitative knowledge of AGB winds is still poor and forces us to adopt parametric treatments, where observations play a crucial role in fixing the (otherwise free) parameters (Wood 2003; Olivier \& Wood 2003; Wood et al. 2004; Andersen et al. 2003).

Similar problems affect the estimates of the stellar luminosity. Observations are hampered by the difficulties of measuring the distances for single, often obscured objects like AGB stars. On the other hand, the luminosities derived from full stellar evolutionary models are affected by the uncertainties in the choice of the mixing parameters (in particular of the extension of convective overshoot) and of surface atomic and molecular opacities (Marigo et al. 2003). Models adopting large overshoot parameters (see e.g. Izzard et al. 2007) derive large values for the mass dredged-up after each thermal pulse, thus obtaining the surface enrichment in $\mathrm{C}$ and $s$-elements earlier and at a lower luminosity than models based on the Schwarzschild's criterion can do.

In a previous paper of this series, hereafter referred to as "Paper I" (Guandalini et al. 2006), we analyzed a sample of C stars reconstructing their SEDs up to far infrared, on the basis of space-borne infrared observations from the ISO and MSX missions. We found evidence for a relatively large average $\mathrm{C}$-star luminosity, thus suggesting that the so-called " $\mathrm{C}$-star luminosity problem" (Cohen et al. 1981) might not be real, being simply an effect of poor estimates of the luminosity, due to insufficient knowledge of the mid-infrared emission. We also reviewed the available mass loss rates and showed their correlation with infrared colors.
We want now to extend that analysis, considering those thermally-pulsing AGB stars where the enhancement of C (and $s$-elements) is more moderate than in C stars: it is the case of MS and S giants (Busso et al. 1992, 1995). In Sect. 2 we present the sample stars, and we discuss the choices made in selecting and organizing them in sub-samples, according to the quality of the available data. In Sect. 3 we present the IR colors and derive the bolometric corrections, based on a set of sources whose magnitude can be estimated safely through the integral of detailed SEDs. We also use these corrections for inferring the critical parameters (absolute magnitudes or distances) of sources for which either i) we have incomplete IR coverage but reliable distance estimates; or ii) period-luminosity relations yield the luminosity, and the distance needs to be inferred from the distance modulus. (For the sake of clarity, the adopted period-luminosity relations are discussed in Appendix A.) Once the absolute magnitudes are known, in Sect. 4 we can analyze HR diagrams and luminosity functions and on this base we also attempt a rough estimate of photometric parallaxes for Mira variables with no other available data on luminosities. Then, in Sect. 5 some preliminary conclusions are derived, while we postpone to a forthcoming dedicated work the analysis of stellar winds.

\section{The sample of S stars}

We started from the extended lists by Stephenson (Stephenson 1984, 1990), containing O-rich evolved red giants with known or suspected "S star-like" chemical peculiarities. As mentioned, these last anomalies can be due either to in-situ dredge-up of newly produced elements (intrinsic-S stars) or to mass-transfer episodes in a binary system (extrinsic-S stars). From those catalogues we selected a total of 613 sources for which measurements in the near-IR are available (from 2MASS, see Cutri et al. 2003). Another required property for selection was the existence of mid-infrared photometry (although with varied detail and spectral coverage). The chosen sources fall into various categories, depending on the extension and quality of the information we could collect on their IR colors, distance, variability type, period, luminosity. As a result, we can organize the stars selected in the following four sub-samples:

- Sub-sample A. This contains 21 sources for which ISO-SWS measurements exist up to long wavelengths (40-45 $\mu \mathrm{m})$, so that a detailed SED can be obtained. The mid-IR SEDs can then be integrated together with near infrared photometric points, in order to infer a very reliable (apparent) bolometric magnitude, by means of the relations of fundamental photometry (Glass 1999; see also Paper I). In this way we have a means for computing bolometric corrections and look for their correlations with available parameters. Much like for C stars (Paper I), these bolometric corrections are correlated to near-to-mid infrared color indexes. About $50 \%$ of the stars in sub-sample $A$ have also astrometric estimates of the distance from the revised Hipparcos catalogue (van Leeuwen 2007a,b); for some others we could infer a distance from the variability period. In all such cases, also the absolute magnitudes can be obtained: the stars with these characteristics are therefore the fundamental bricks on which we build the rest of our work. In particular, bolometric corrections offer us the tools for deriving absolute bolometric magnitudes for AGB stars outside this main sub-sample $A$, whenever observations of at least one near-to-mid color index exist. 
- Sub-sample $B$. Here we put all sources (21) for which a reliable estimate of the distance is available (e.g. from Hipparcos, especially after the recent revision by van Leeuwen 2007a,b), but a detailed mid-infrared SED is not present. Although the stars of sub-sample $B$ have only sparse mid-infrared photometry (from MSX, IRAS-LRS or ground-based measurements), thanks to the bolometric corrections discussed above absolute bolometric magnitudes can be computed for all of them.

- Sub-sample $C$. This class contains 41 Mira-type stars, for which near-to-mid IR colors could be derived from either MSX or IRAS measurements. Their Mira variability, of known period, allows for the application of periodluminosity relations, yielding the absolute luminosities (see in particular Whitelock et al. 2008). Some comments on the procedure used in this case to homogenize period-luminosity relations are presented in Appendix A. By also applying the bolometric corrections computed from sub-sample $A$ to their photometric data, the apparent bolometric magnitudes can be derived, so that an estimate for the distance can be inferred from the distance modulus.

- Sub-sample $D$. Here we collect all the remaining sources $(\sim 500)$. One or a few measurements at mid-infrared wavelengths exist also for this sub-sample; these data can be coupled to 2MASS photometry, giving near-to-mid infrared colors on which to apply the bolometric corrections. In this way, we can at least derive reliable bolometric (apparent) magnitudes. As the distance is in general not known from measurements (unlike for sub-sample $B$ ), and neither it can inferred from any known period (unlike for sub-sample $C$ ), a precise value for the absolute magnitude cannot be given at this stage and will have to wait for estimates of the distance. However, we shall see that, at least for known Mira variables, fiducial intervals for the distance can be derived from the HR diagram and the luminosity functions, in the form of a rough photometric parallax.

The first three samples, together with the input data we shall analyze for them, and the resulting estimates for their fundamental parameters are placed in the "online material" as Tables 1 to 9. The tables of the last sample are available electronically at the CDS.

In general, we perform the same kind of analysis already presented in Paper I for C-rich AGB stars, computing the bolometric corrections and bolometric apparent magnitudes, the absolute magnitudes when possible, and expressing the color data in the photometric system described in Paper I and in Busso et al. (2007), hereafter Paper II. In order to adopt this photometric system, we need to perform a re-binning of space-borne spectroscopic data, by convolving them with the response of the filters. We then discuss luminosities and luminosity functions for understanding the evolutionary relations between $\mathrm{S}$ stars and $\mathrm{C}$ stars along the thermally-pulsing AGB.

\section{Infrared colors and bolometric corrections}

We have made a preliminary control that the choice of near and mid IR wavelengths is sufficient for our scope. In fact, one might a priori believe that the inclusion of optical bands could increase the accuracy of bolometric corrections (despite the large uncertainties related to the photospheric variability). However, for all the stars of our samples for which we could find archived visual magnitudes, the optical-to-near-IR colors (e.g. $V-J$ ) are always in excess of 3 mag. Most $V-J$ colors lay in the range 4-7 and a few in the range 7-10 mag, implying flux ratios $f_{J} / f_{V}$ larger than at least a factor of 10 and more often a factor of 100 . There would be therefore no significant change in the results by introducing optical photometric data. S stars are certainly bluer than $\mathrm{C}$ stars (only a couple of sources are dominated by mid-IR, as is instead common for C-rich Miras, like CW Leo); but even when the infrared excess by dust is minimal and the photospheres are optically bright, all AGB stars, including S stars, remain mainly IR objects. The bluest of our sources have maximum emission in the $H$ band.

As discussed in the previous section, we then start from the sources of sub-sample $A$ in order to derive the bolometric corrections, then proceed to the other stars in our selection. The relevant parameters we collect or derive can be divided into three categories, so we have three tables per each sub-sample.

Table 1 presents the general characteristics of sub-sample $A$. This includes: i) the names of the sources (from IRAS, from the Stephenson's compilation (Stephenson 1984, 1990) and, when available, from the variable star nomenclature); ii) the coordinates (with the equinox fixed at year 2000.0) as given by the ICRS on the SIMBAD database; iii) the spectral types; and iv) the variability type, according to the General Catalogue of Variable Stars (GCVS, Samus et al. 2004). Some of the stars we analyze have uncertain spectral classifications for various reasons. As this point is not among the main issues we want to address, in all these cases we indicate the alternative choices found in the literature, simply separating them by a slash $(/)$. In some cases, our analysis will yield suggestions on the correct classification.

The ISO-SWS SEDs of a few sources of group $A$ are shown in Fig. 1, organized according to their spectral classification (MS or S) and variability type (Mira, Semiregular or Irregular). The figure shows one of the most evident properties of AGB stars, present also in C- rich sources: the Mira-type variability is often associated with a remarkable IR excess and a SED peaked at longer wavelengths than for Semiregular or Irregular variables. As compared to $\mathrm{C}$ stars, however, the IR excess of $\mathrm{S}$ stars is in general much smaller, as we already mentioned. This is probably related to intrinsic properties of dust with O-based or C-based composition. A similar difference exists for molecules (e.g. gaseous component) of the two species, as seen from the photospheres, as we shall argue later.

The above very detailed energy distributions are integrated to estimate the apparent bolometric magnitude, using the relation:

$m_{\mathrm{bol}}=-2.5 \int_{0}^{\infty} f_{v} \mathrm{~d} v+C$

as discussed by Glass (1999), who also gives $C=-18.98$ in the case where the total flux is expressed in $\mathrm{W} \mathrm{m}^{-2}$. This relation also provides the zero-magnitude flux. From Eq. (1) one obtains:

$f_{0}=\int_{0}^{\infty} f_{v} \mathrm{~d} v=1.94 \times 10^{-7} \mathrm{~W} \mathrm{~m}^{-2}=1.94 \times 10^{19} \mathrm{Jy} \mathrm{Hz}$.

Following the procedure described in Papers I and II, we again operate a re-binning of the ISO spectra to derive estimates for the fluxes in a number of broader-band infrared filters (with $10 \%$ bandwidth), in the range between 8 and $14 \mu \mathrm{m}$ (centered at $8.8,9.8,11.7$ and $12.5 \mu \mathrm{m}$ ). (The fluxes in such filters are usually indicated as the equivalent wavelengths in normal brackets; the magnitudes use a similar notation but with squared brackets). These filters are of rather common use in ground-based infrared cameras; our choice is aimed at making the space-borne measurements more easily comparable with present and future 

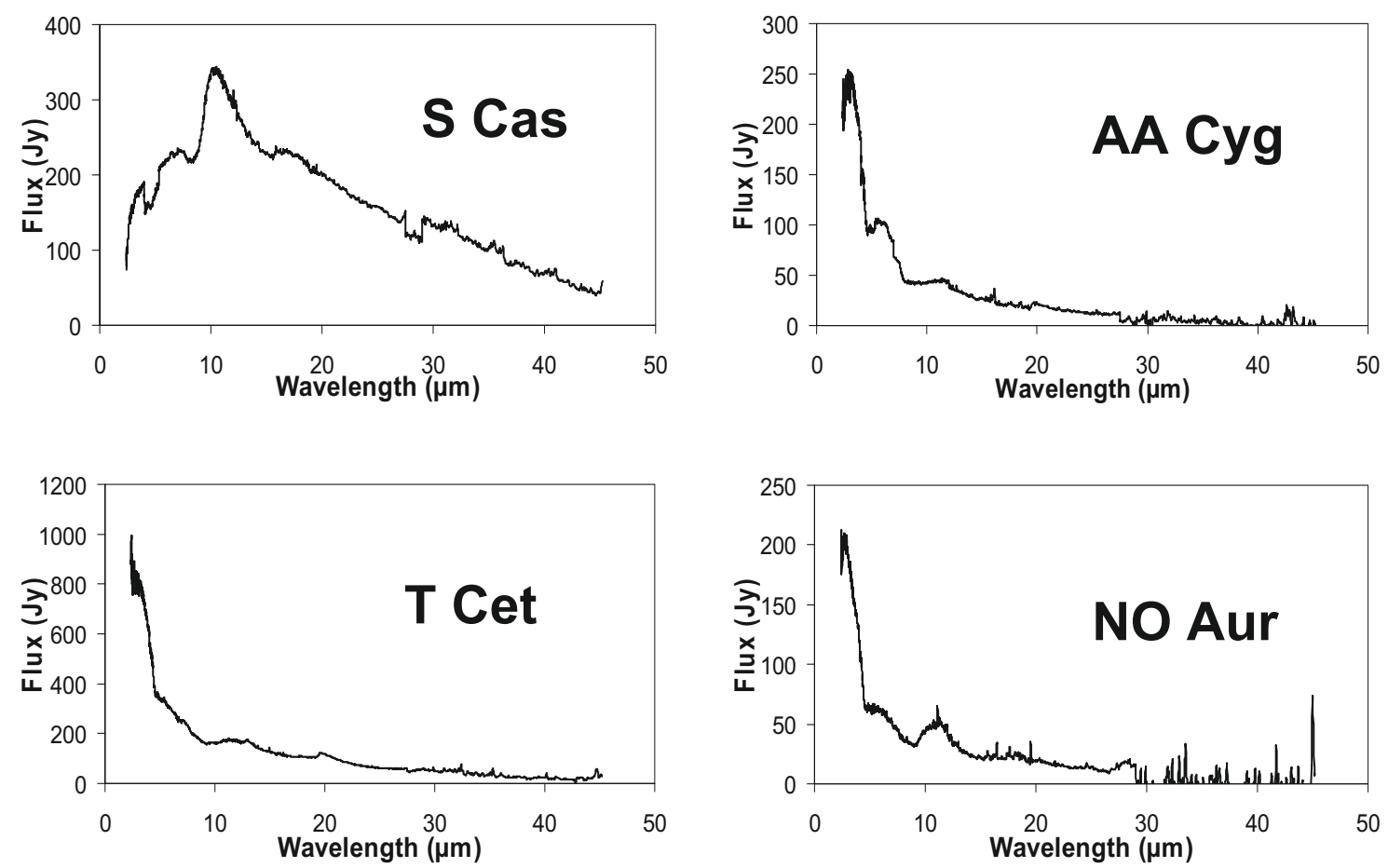

Fig. 1. Examples of ISO-SWS spectra of AGB stars. Upper left: a S-type Mira variable (S Cas). Upper right: a S-type Semiregular source (AA Cyg). Lower left and right: two MS stars.

photometric observations and to infer for these last suitable bolometric corrections. At long wavelengths $(\lambda>14 \mu \mathrm{m})$ the re-binning is made using the response curve of the MSX filters $D(14.6 \mu \mathrm{m})$ and $E(21.3 \mu \mathrm{m})$. Zero-magnitude fluxes (in Jy) for our chosen photometric system are: $52.23(8.8 \mu \mathrm{m}), 42.07$ (9.8 $\mu \mathrm{m}), 29.55(11.7 \mu \mathrm{m}), 25.88(12.5 \mu \mathrm{m}), 20.25(14.6 \mu \mathrm{m})$, $8.91(21.3 \mu \mathrm{m})$. For near-IR, 2MASS calibrations for the $J, H$ and $K_{\mathrm{s}}$ filters are given in Cohen et al. (2003). (Hereafter we shall adopt for the $K$ "short" $K_{\mathrm{s}}$ filter the simple notation $K$.)

Figure 2 gives some examples of the SEDs computed in the photometric system thus defined, for the various types of AGB stars discussed in this note. The same data are listed in Tables 2, 5 and 8, together with near-infrared fluxed obtained from the 2MASS catalogue.

As a consistency check, we repeated the calculations of Eq. (1) using the apparent bolometric magnitudes previously derived from ISO spectra, and making the integral over the low-resolution SEDs of Fig. 2. By adopting the zero-magnitude fluxes by Bessell et al. (1998), we obtain for the integration constant an average value $C=-19.0$. The fact that this estimate be very close to the real $C$ value given above certifies that the our re-binning is done properly, and does not alter in any significant way the integrals of Eq. (1), hence the magnitudes of our stars.

Once the apparent bolometric magnitudes are known, for those sources for which a reliable estimate of the distance exists we can derive $M_{\text {bol }}$. This estimate can be obtained also for those Mira variables of sub-sample $A$ for which an astrometric measurement of the distance is not available, but the variability period is known. Indeed, the period-luminosity relations of Mira stars have become sufficiently reliable that we can use them to infer $M_{\text {bol }}$ and then obtain the distance from the distance modulus (see Appendix A). The limit of this technique lays in the fact that, not having a proper description of the interstellar extinction, we are forced to exclude the extinction correction. The error introduced on infrared colors, however, is not large, and the related uncertainty remains within the (broad) observational errors of typical infrared photometry (see Paper I for a discussion).

Table 3 presents the absolute bolometric magnitudes derived for sub-sample $A$, with the indication of the method used, i.e. period-luminosity relations (labeled "this paper") or distances in the literature (labeled after the reference adopted, either "Hipparcos", see van Leeuwen 2007a; or in a single case "Groenewegen", taken from Groenewegen et al. 2002). The values adopted for either the period or the distance are also indicated.

We can then correlate the newly found bolometric magnitudes with color indexes in the infrared, in order to infer bolometric corrections to be applied to the other groups of stars in our sample. In Fig. 3 we present two of the most significant such correlations. They are shown as corrections to a mid-infrared magnitude ([8.8] or [12.5]) as functions of near-to-mid infrared color indexes $(K-[8.8]$ or $K-[12.5]$ respectively). Least-square fits, shown in the panels of the figure, correspond to:

B.C. $=-0.0118 \cdot(K-[8.8])^{2}+1.1552 \cdot(K-[8.8])+2.3061(3)$ and to

B.C. $=-0.0195 \cdot(K-[12.5])^{2}+1.1949 \cdot(K-[12.5])+2.1918(4)$

with correlation coefficients of $R=0.990$ and $R=0.993$, respectively.

As shown in the figures, both relations are quite tight and offer a reliable way to estimate absolute bolometric magnitudes for stars for which we have only partial photometric coverage. In the two plots of Fig. 3, there is only one point which is significantly discrepant and does not follow the average correlation. Quite significantly, this point corresponds to II Lup, a star that, although listed in the Stephenson's compilation of S stars, is instead a carbon-rich Mira variable and must therefore be excluded in discussing the photometric properties of O-rich AGB stars. 

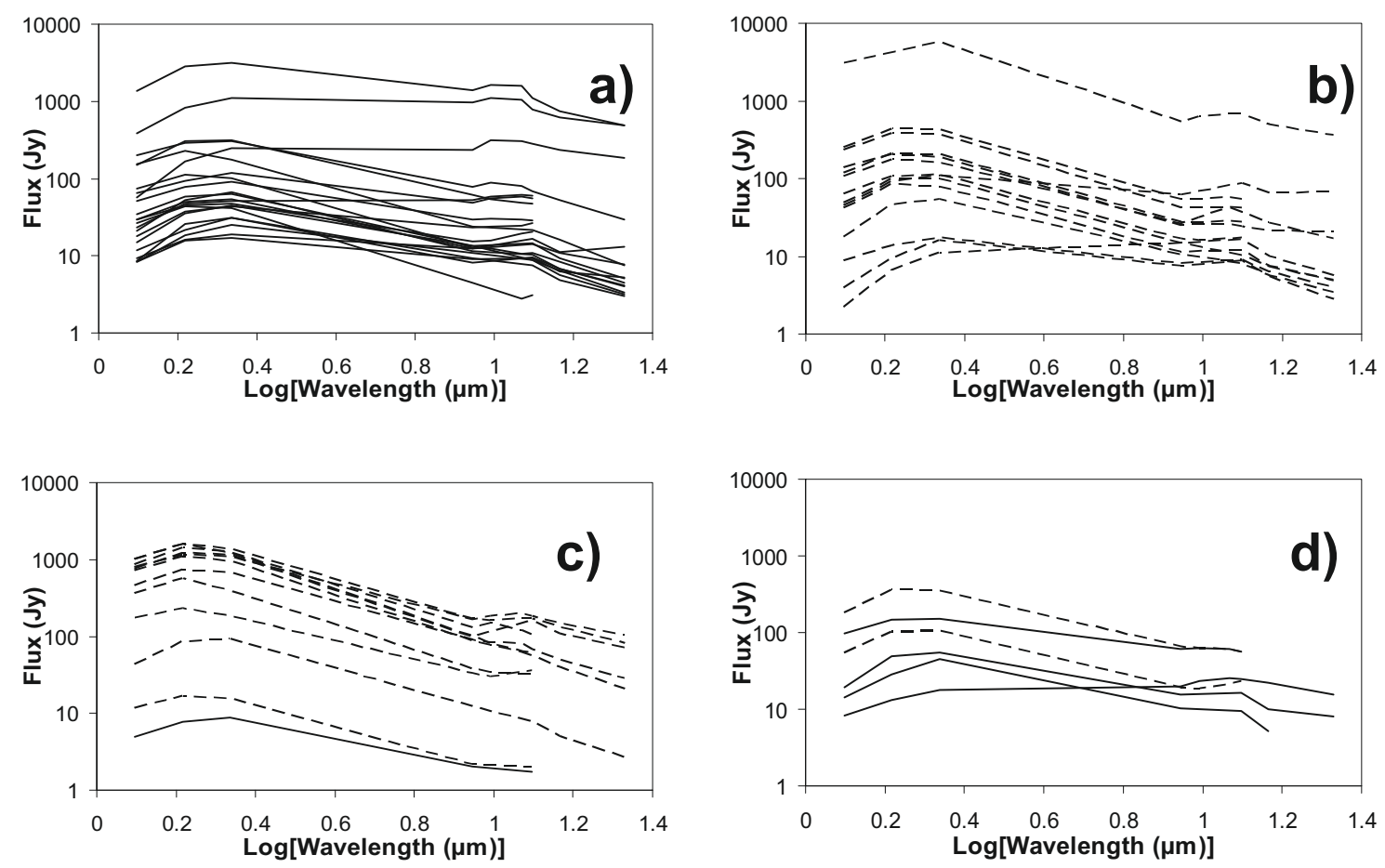

Fig. 2. Spectral energy distributions of sample stars after rebinning in the photometric system of Paper I. a) S Miras. b) S Semiregulars. c) MS sources. d) SC stars. Continuous lines refer to Miras, dashed ones to Semiregulars.
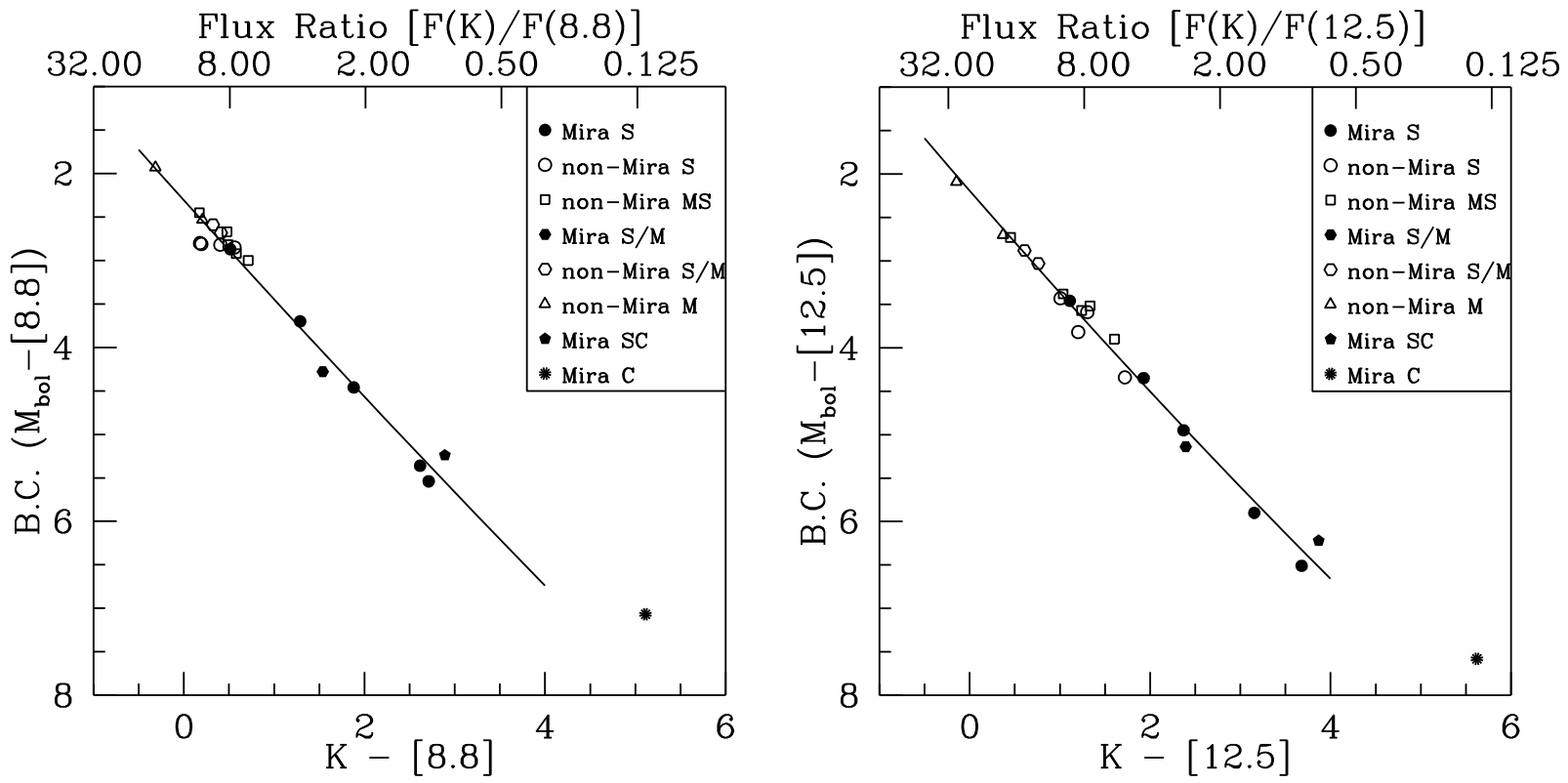

Fig. 3. Two examples of bolometric corrections, suitable for MS-S Stars with infrared photometry available. Correlation coefficients for the two relations are $R^{2}=0.98$ (left) and $R^{2}=0.99$ (right). Here and elsewhere " $K$ " is a compact notation for the 2MASS filter $K_{\mathrm{S}}(K$-short).

At this point we need to underline a relevant characteristic of the data displayed in Fig. 3. The corrections there used to infer the bolometric emission are applied to mid-infrared magnitudes, i.e. to data that, for our S stars with moderate IR excess, are essentially constant. This constancy of the mid-IR emission is important for estimating better bolometric corrections and is not shared by very red objects, e.g., by the carbon-rich Miras, where also the emission from the circumstellar envelope varies significantly in time (see Paper II). Anyhow, for our sample stars in Fig. 3 we expect roughly constant magnitudes at 8.8 or $12.5 \mu \mathrm{m}$, while the $K$ (short) estimates from 2MASS, being single-epoch measurements, are affected by the photospheric variability (that can be up to $1-1.5$ mag in near-IR). Different stars were certainly (randomly) sampled by 2MASS in different epochs of their light curves, but despite this, all the data fit a single regression line quite well. This can be so only if, during a cycle, the representative points of our stars move along the regression line itself. One might wonder why. The variability of AGB stars is not necessarily related only to a transfer of energy from one wavelength range to another (due to the star becoming cooler and more extended or hotter and more compact). There might be actually a global variability of the bolometric 
magnitude itself, because we can see the addition, with different percent weight, of energy from finite-amplitude mechanical oscillations and shock waves affecting the envelope. The color dependence of these emissions is unknown to us, but we know experimentally that it does not extend to mid-IR for moderately red objects. If the chosen mid-IR magnitudes remain essentially constant, then bolometric corrections variable in time will be needed to compensate for the near-IR (and for the possible bolometric) variability. When the star is brighter it will require a larger correction, when it is dimmer the correction will be smaller, but as long as the regression line remains valid, the given formula for the B.C. should continue to hold. This would not be true if we had chosen, in the ordinate, a near-IR magnitude, still affected by the photospheric variability, having an unknown color dependence.

It remains however true the bolometric magnitudes given here, although possibly rather well estimated thanks to the use of mid-IR data, might be intrinsically variable. We have estimated how much the rather common variability by about 1 mag in $K_{\mathrm{S}}$ would affect the global flux, and this can reach up to $25 \%$ of the total. When estimating absolute bolometric magnitudes, throughout this paper, we apply both the corrections of Fig. 3 whenever possible, and then take the average of the absolute magnitudes thus obtained (the values to be averaged are in any case very close to each other).

We then applied the above corrections to the IR colors of sub-sample $B$, thus deriving also for them an estimate of the absolute bolometric magnitude. The input data and the derived parameters for this sample are shown in Tables 4 to 6.

A somewhat inverse procedure is applied to the sources of sample $C$, where luminosities can be inferred from periodluminosity relations. Here a comparison with the apparent bolometric magnitude, derived from bolometric corrections, yields the distance of the object. The recent updates in periodluminosity relations for AGB stars, which now allow for this possibility, are discussed in Appendix A.

The input data and the derived parameters for sample $C$ are illustrated in Tables 7 to 9. The whole (more sparse) information we could compile for the bigger but so far incomplete subsample $D$ is included instead in Tables 11 and 12, which we publish in electronic form at the CDS due to their dimensions. For the content of Table 10 see next section.

\section{HR diagrams and luminosity functions}

Using the IR fluxes collected in the previous section, and the information acquired on the absolute luminosities (see also Appendix A), we can now study the photometric properties of $\mathrm{S}$ stars and their relatives, with the aim of understanding how they are linked to the evolutionary status of the sources and to their chemical properties.

Figure 4 illustrates the $J-K$ vs. $K-[8.8]$ color-color diagram of the sources in our sample. The variability types appear rather well discriminated from IR colors. With only one exception (an SC star believed to be of Semiregular variability, but that we suspect is instead a misclassified Mira), all Semiregular and Irregular variables lay in the lower-left part of diagram, and remain separated by Miras by a dividing gap (the dashed line is just an eye's guide to illustrate this). Similarly, only very few objects classified as Miras fall at the left of the guiding line, all of them with short periods, while the vast majority is, in both colors, redder than Semiregulars and Irregulars. This is not surprising, in view of the fact that Miras are more efficient mass-losers and, as we argue below, are on average more evolved. We actually

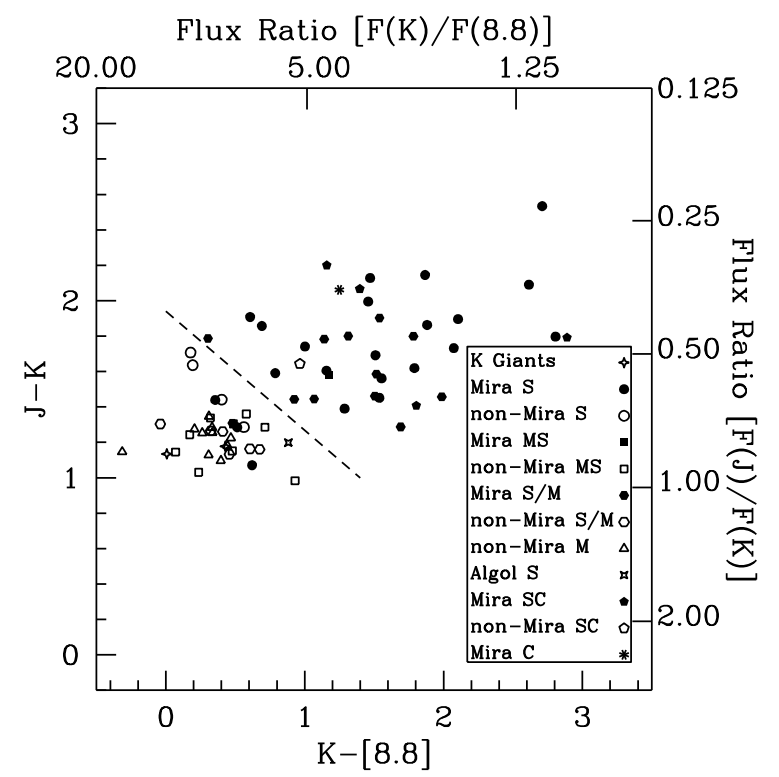

Fig. 4. A color-color diagram of the sources in samples $A$ to $C$. All of them belong to the Stephenson's list, but some are misclassified: indeed, we find a few $\mathrm{K}$ and $\mathrm{M}$ giants, and a couple of $\mathrm{C}$ stars. Mira variables are redder than other sources in both colors and generally well separated from the rest by IR color criteria.

interpret the emerging evidence (from both the present sample and those of Papers I and II) as a suggestion that efficient radiation pressure on dust grains, powering fast mass lost, starts when the star has reached the Mira-like variability at periods in excess of about 200-230 days. In any case, the use of IR colors for discriminating different sources is interesting because the separation of the types is quite good, to the point that the IR colors might be used for guessing the variability type. (For example, if one picks up an AGB star of unknown variability, with $J-K$ and $K-[8.8]$ colors near 2 , then we can reliably assume that it must be a Mira star.)

Figures 5 and 6 show two examples of the absolute H-R diagrams derived from our sample stars (the figures refer to both intrinsic and extrinsic sources). They are presented as a function of $J-K$ and $K-$ [8.8] colors, which can be considered as monitors either of the photosphere alone $(J-K)$ or of the inner circumstellar environment ( $K-$ [8.8]). The link between fluxes in Jy and magnitudes in the various filters, hence to colors, is established by i) the distance correction (no extinction is assumed): and ii) the calibration of zero-magnitude fluxes given above.

The HR diagrams contain a large number of useful pieces of information on S star morphology and physics. We identify here a few such issues.

- Absolute magnitudes of Semiregular and Irregular variables stay on an almost vertical sequence, close to the region covered by AGB stellar models, albeit with remarkable scatter. Below this sequence, at the bottom of the plot, we find sources whose luminosities suggest that they are in an evolutionary stage preceding the thermally-pulsing AGB. These must necessarily be extrinsic S or MS stars; indeed, in most cases their extrinsic nature is already well established. For those sources (five) in this group for which no information on binarity is available, we suggest here, as a result of our luminosity calibration, that they are extrinsic and not yet on the TP-AGB (see the relative indication in bold and underlined in Tables 3, 6, 9). This suggestion will now need independent confirmations, which would also indirectly verify 


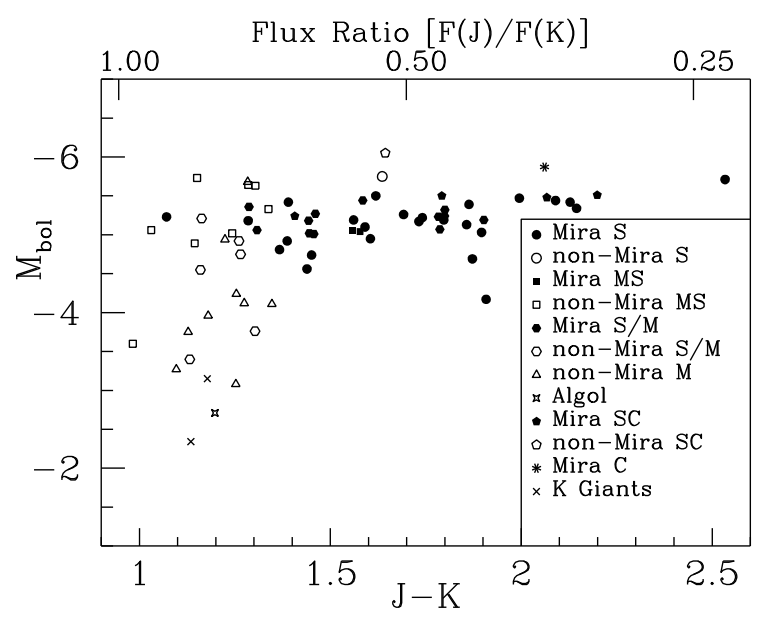

Fig. 5. An example of an absolute HR diagram, built using a near IR color index as abscissa.

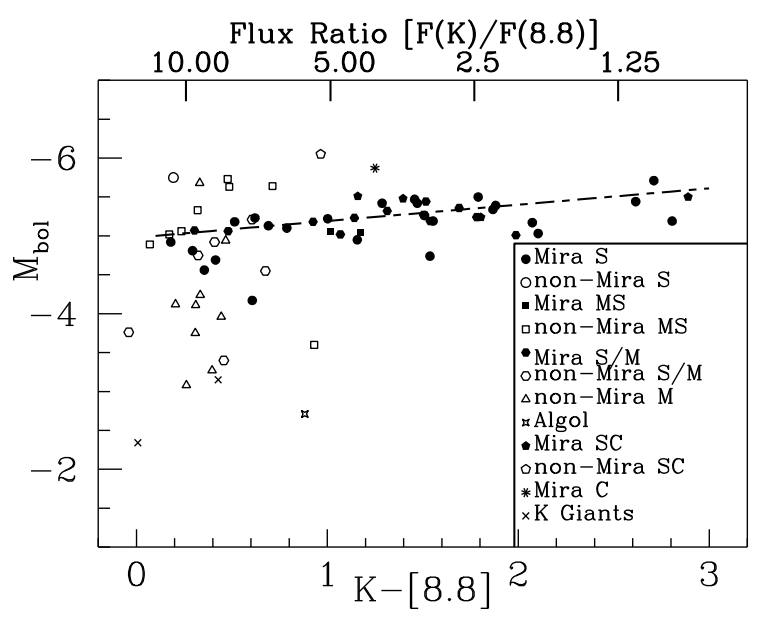

Fig. 6. An example of an absolute HR diagram, built using a near-tomid IR color index as abscissa.

(or deny!) the validity of our bolometric corrections. If we are right, then extrinsic S stars should be the O-rich equivalent of the carbon rich R stars; Fig. 5 supports this guess in showing that lower-luminosity $\mathrm{S}$ stars are also warmer than the others.

- S-star infrared colors are different from those of C stars, being on average much bluer. Infrared excesses are less extreme, although mid-infrared (especially the [8.8] filter) remains the crucial wavelength range for understanding their physics: in particular we underline that, also for $\mathrm{S}$ stars, the best relations determining bolometric corrections are obtained by making use of mid-infrared colors.

- The un-reddened MS-S sources distributed along the vertical branch in Figs. 5 and 6 are more compatible with the evolutionary AGB tracks than for similarly-positioned C stars. Red-shifts of the track, requiring temperature corrections (and indicating inadequacy in molecular opacities of the envelope), although present, are largely reduced (by 0.5-1 mag) as compared to C stars (see Paper II).

- Absolute magnitudes of Mira variables are distributed over a relatively well defined distribution. They show a linear trend, indicating higher luminosities for redder colors and the correlation is clear, although the slope is small. In Fig. 6 we have added a least square curve based only on bona-fide, intrinsic S Miras. The relation is:

$M_{\text {bol }}=-0.2104 \cdot(K-[8.8])-4.9765$

with a correlation coefficient $R=0.78$. Of course one expects different luminosities for sources of different colors (period-luminosity relations are such that the LPVs of longer period are also redder). It is however interesting to notice that, despite the infrared excesses increasingly separate the observed points from the AGB model tracks, we can nevertheless determine a systematic trend in the magnitude (now dominated by the circumstellar envelope). Moreover, it's remarkable that the correlation is sufficiently tight not to be confused by the observational errors.

If one computes a global average of the absolute magnitudes of Miras, this turns out to be $-5.15 \pm 0.4$. Once stars have reached this luminosity range and are in the Mira variability stripe, further remarkable increases of luminosity seem to be no longer possible. This limiting range of luminosities (less than 1 mag wide) is close to a similar one that can be inferred for C-rich Miras in the data of Paper I. Since the range is relatively small, Mira variables appear to have, on average, similar Magnitudes, independent of the chemical composition of the atmosphere.

- We can confirm for S stars what was already said for C-stars: the Mira variability type tends to occur primarily at the end of the evolution. It was argued in the past that Semiregulars might be AGB stars in the low-luminosity post-flash dip (Kerschbaum \& Hron 1996), so that a repeated transition between the two main variability types was expected. We cannot exclude this in general, but the high statistical relevance of Semiregular variables (almost as abundant as Miras in our global database, including sources of group $D$ ) cannot be explained by the post-flash phases, which, for advanced thermal pulses, occupy at most $25 \%$ of the TP-AGB duration (see also Fig. 8).

- The limited spread of Mira absolute magnitudes and the sufficiently good correlation with IR colors offer a tool for obtaining a first-order approximation to the luminosity and distance of Mira stars for which we do not have information beyond the measured colors. Indeed, for them one can first derive the apparent bolometric magnitude from our bolometric corrections; then one can assume, as a reasonable guess, that bolometric absolute magnitudes have an average of $-5.15 \pm 0.4$ and a correlation with the $K-[8.8]$ color like the one shown for Fig. 6 . This allows a determination of the distance from the distance modulus. An example of the application of this procedure to Mira variables in sample $D$ is shown in Table 10.

- Stars differently classified along the sequence MS, S, SC show a different behavior also for what concerns the variability. Albeit with some scatter, one can notice that the MS classification is in general (with two possible exceptions) accompanied by the Semiregular or Irregular variability types, while a much larger percentage (about $60 \%$ ) of S stars shows the Mira-type variability. Concerning SC stars, of the $5 \mathrm{ob}-$ jects for which we have detailed information, 4 are of Mira type; from the color-color diagram of Fig. 4 we suspect this should be so also for the fifth member, for which we suggest that the previous classification as a Semiregular might be wrong. SC stars are in general red, cool and luminous, like most S Miras. They might be the final evolutionary status of stars whose mass is barely sufficient to dredge-up enough carbon to approach unity in the $\mathrm{C} / \mathrm{O}$ ratio. 


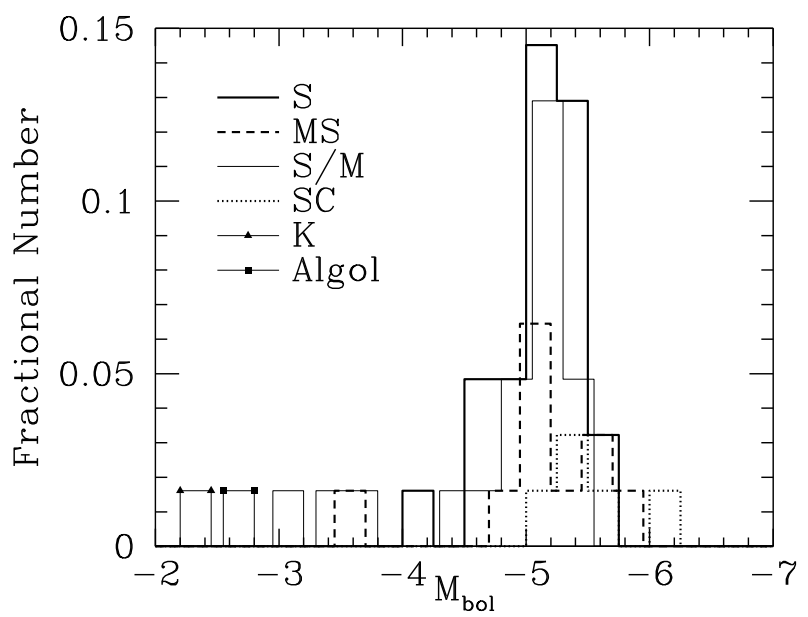

Fig. 7. The luminosity function of the stars in our samples $A$ to $C$. Low luminosity sources are always extrinsic and the $S$ stars define a very narrow distribution, characterized by a magnitude $-5.15 \pm 0.4$.

The luminosity properties illustrated by the HR diagrams, and in particular the narrow range over which the magnitudes of MS-S Miras are distributed, are illustrated by the histogram of Fig. 7, showing the luminosity function of our sources. The distribution has a very well defined peak: all MS, S, SC stars lay in the range -4 to -6 and Mira $S$ stars occupy a thin slice across the magnitude values -4.8 to -5.5 . All the data points significantly far from this peak refer to extrinsic sources not belonging to the TP-AGB phase. Many (most) sources of uncertain classification (so far indicated as S/M) fall in the fiducial interval of the "best" $\mathrm{S}$ stars. We are pretty sure they are indeed S stars and probably Miras. In Tables 3, 6, 9 and 12 we indicate the possible classification deduced from Van Eck et al. (2000); Yang et al. (2006). In a few cases of discordant indications we prefer the physical analysis by Van Eck et al. (2000) and we show the other in parenthesis. For five sources in Tables 6 and 9, for which neither study offers a suggestion, we can infer that they are extrinsic from their low intrinsic luminosity.

Like for C stars, and actually even more so, the luminosities of Population I S stars are well defined inside a narrow range and are high enough that normal stellar models based on the Schwarzschild criterion for convection can adequately explain their formation. Once again, and as already noticed in Paper I, stellar models using large convective overshoot to favor dredge-up, and to obtain S-star and C-star chemical peculiarities at low luminosities (Izzard et al. 2007) appear to be unjustified, at least as far as the AGB evolutionary stages are concerned. Recently Bonačić Marinović et al. (2007) suggested that such models should be preferred for their capability of reproducing $s$-process abundances in stellar populations. One has however to mention that, from a theoretical point of view, the several free parameters still involved in any modelling probably make any conclusion provisional, and require observational verifications. Infrared observations, as presented here, do not confirm the need for large overshooting, at least for what concerns the ensuing stellar luminosities.

\section{Conclusions}

In this paper we have presented a reanalysis of the properties of MS-S-SC stars, based on a sample of about 600 sources, whose infrared fluxes from 1.25 to $21 \mu \mathrm{m}$ were measured by the 2MASS, IRAS, ISO and MSX experiments. A "best" group of 21 stars (for which detailed ISO-SWS spectra are available up to long wavelengths) allowed us to obtain the bolometric magnitudes from an effective integral of the spectral energy distribution up to $45 \mu \mathrm{m}$. Correlations with near-to-mid IR colors then allowed us to infer bolometric corrections suitable to be applied to other groups of sources, with a less detailed coverage of the energy distribution. We can thus estimate with sufficient accuracy the apparent bolometric magnitudes of more than 500 sources. The whole analysis was performed in the photometric system suggested in Busso et al. (1996) and subsequently used in Papers I and II.

Criteria for obtaining the distance have then been discussed, from the simple use of revised astrometric measurements to a reformulation of the known period-luminosity relations for O-rich long period variables. The results of our analysis suggest that Mira variables of the $S$ type have on average magnitudes in the range $-5.15 \pm 0.4$, showing a well-defined linear correlation with infrared colors, especially the $K-$ [8.8] one. Low-mass AGB stars do not appear to proceed beyond the upper limit of the Mira luminosity range. Inside this range $P-L$ relations have become rather tight and accurate; they now form a very useful tool for determining intrinsic stellar parameters. From the $P-L$ relations and the $M_{\mathrm{bol}}-(K-[8.8])$ relation of Eq. (5) we now have tools to estimate the absolute magnitudes, hence the distances, of Mira S variables for which either the period or the infrared colors have been determined.

From statistical considerations it is also argued that Mira variables should occupy mainly the final part of the AGB track, as the simple intermittency between low-luminosity post flash dips and high-luminosity $\mathrm{H}$-shell-powered stages is not sufficient to explain the available numbers of Semiregulars and Miras.

Luminosity functions confirm that intrinsic S stars (especially if in the Mira class) are distributed over a narrow range around the above average magnitude, and that this last datum is very close to the one previously found for $\mathrm{C}$-stars. This is so to the point that it appears unlikely that a single AGB star can follow the whole M-MS-S-SC-C sequence, by simply increasing gradually its content of carbon and heavy elements as time passes, new dredge-up episodes enrich the envelope and the luminosity increases. Most probably, small differences in the initial mass and metallicity, almost indistinguishable once on the AGB, determine the final chemical fate of a star, which, for increasing initial mass, can end its life either as a MS-S giant, or a SC giant, or reaching effectively the $\mathrm{C}(\mathrm{N})$ stage. As an example of the effects of small mass (and metallicity) differences, Fig. 8 shows two AGB luminosity sequences obtained from the FRANEC code by Busso et al. (2003). The model star of the left panel does not reach the C-star phase, ending as an MS/S star. The evolutionary phases during which S-type chemical peculiarities are exhibited by the envelope are shaded in the plot. In the right panel we instead show the results of a model producing a real C-star: it has a slightly higher mass. Given the magnitudes we found in this paper for the sources of types MS and S, and those found in Paper I for C stars, most of the objects in our sample should belong to the mass and metallicity range covered by Fig. 8. If we broadly consider the typical magnitudes of $\mathrm{S}$ and $\mathrm{C}$ stars as being in the range $-5.2 \pm 0.4$, then it is clear that this range includes both the S-star phase in the first panel of Fig. 8 and the $\mathrm{S}$ - and $\mathrm{C}$-star phases in the right panel. It would be very difficult or impossible to distinguish between the two cases on the basis of their magnitudes, given the remaining uncertainties on distances and on possibly variable bolometric magnitudes. 

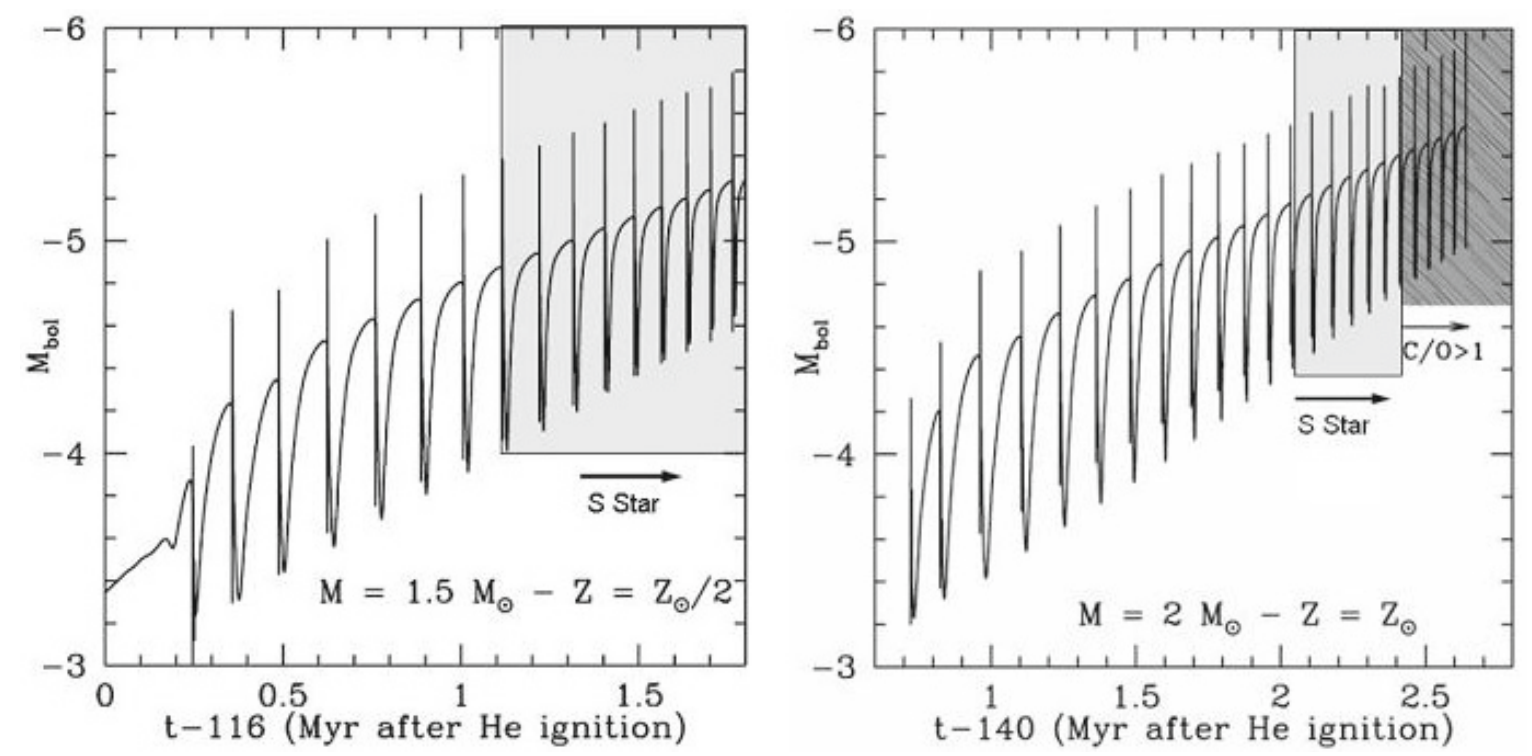

Fig. 8. The magnitude variations of two model stars of Population I, during the thermally pulsing AGB phase, as computed by the FRANEC code. The abscissa shows the time passed after the ignition of He-burning in the core, in Million years (once an offset is deduced, whose value is indicated). Most sources with features typical of $\mathrm{S}$ or $\mathrm{C}$ stars and magnitudes in the range $-5.2 \pm 0.4$ should belong to the mass and metallicity range illustrated here, but only the star in the right panel does achieve the $\mathrm{C} / \mathrm{O}>1$ condition.

The above results, and the typical initial mass implied for $\mathrm{S}$ and C stars $\left(1.5-2 M_{\odot}\right)$ might appear rather peculiar, in view of the fact that about half of Planetary Nebulae are carbon rich. This cannot be explained by progenitors of around $2 M_{\odot}$, at least according to the most common choices for the Initial Mass Function. However we must remember that the efficiency of dredge up strongly increases with decreasing metallicity. In halo stars one solar mass might be sufficient for forming a $\mathrm{C}$ star (also because the abundance of oxygen is very small). Hence the results presented here should be considered as valid only for galactic disc stars, at relatively high metallicities.

Acknowledgements. We are grateful to the anonymous referee for a very useful, careful and stimulating report, which greatly helped in the improvement of this work. We acknowledge support from the Italian Ministry of Research, under contract PRIN2006-022731, and from the section of Perugia of the National Institute for Nuclear Physics (INFN). This study is also part of the preparatory work for the IRAIT Antarctic telescope, of the Piano Nazionale delle Ricerche in Antartide (PNRA). These studies on Antarctic Astronomy are profiting of the financial support and cultural possibilities offered by the European Coordinating Action ARENA (within the FP6 plan). This research has made use of the SIMBAD database and the VizieR service (CDS, Strasbourg, France), and the IRSA (NASA/IPAC InfraRed Science Archive) database (USA), and the Astrogrid database (UK). In particular archived data from the experiments MSX, ISO-SWS and 2MASS were used. - The processing of the science data of the Midcourse Space eXperiment (MSX) was funded by the US Ballistic Missile Defense Organization with additional support from NASA Office of Space Science. - The Infrared Space Observatory (ISO) is an ESA project with instruments funded by ESA Member States (especially the PI countries: France, Germany, The Netherlands and UK). • 2MASS (Two Micron All Sky Survey) is a joint project of the Univ. of Massachusetts and the Infrared Processing and Analysis Center (IPAC) at California Institute of Technology, funded by NASA and the NSF (USA).

\section{Appendix A: Period-luminosity relation}

Mira stars have been alternatively suggested to be radially pulsating either in the fundamental mode, or in the first overtone (Tuchman 1999; Feast 1999). For Semiregular variables various overtones and the same fundamental mode are also possible. The discussion on these properties has gone on for three decades, although the large database made available by the MACHO project
(Alcock et al. 1992) seems to favor the fundamental mode for Miras (Wood et al. 1999).

The non-unique pulsational properties of Semiregular variables imply that there is not a unique $P-L$ relation for them (Bedding \& Zijlstra 1998). Instead, such relations for O-rich and C-rich Miras have become increasingly accurate and statistically relevant, to the point of forming now an invaluable tool in the difficult task of determining the distances of Long Period Variables. Many studies in the past were dedicated to correlate $\log P$ with magnitudes obtained in the $K$ filter (Feast et al. 1989; Hughes \& Wood 1990; Wood \& Sebo 1996). This method, however, meets the difficulty of the still remarkable light-curve amplitude at $2 \mu \mathrm{m}$. Relations directly connecting the period to the bolometric magnitude (Feast et al. 1989; Whitelock et al. 1994, 2006) are therefore precious, although obviously more difficult to obtain.

Different relations are expected (and found) for C-rich and O-rich objects (see in particular Whitelock \& Feast 2000; Whitelock et al. 2006). For our sample of S-stars, which have $\mathrm{C} / \mathrm{O} \leq 1$, we can use the $M_{\mathrm{bol}}-P$ relation suggested for O-rich Miras by Feast et al. (1989) and Whitelock et al. (1994).

Another formula, established for Magellanic Cloud O-rich Miras, but suitable also for the Galaxy in view of the universality of the slope (Feast 2004), was presented recently by Whitelock et al. (2008). This last relation is based on the $K$-magnitude. It is difficult to establish criteria for choosing between the two, so that we decided to use both, and then take an average of the results. (We underline that both the original relations were derived for $\mathrm{O}$-rich sources and should therefore apply also to $\mathrm{S}$ stars, where the ratio $\mathrm{C} / \mathrm{O}$ does not reach unity.) In particular, our procedure was the following one. i) We firstly derived apparent bolometric magnitudes from the bolometric corrections of Fig. 3, then we applied the relations by Whitelock et al. (1994) to obtain an estimate of the absolute bolometric magnitudes. Hence from the distance modulus we could infer a first value for the distance. ii) Separately, we applied the formula by Whitelock et al. (2008) to derive the absolute $K$ magnitude, then by comparison with the 2MASS measurement we deduced a second estimate for the 


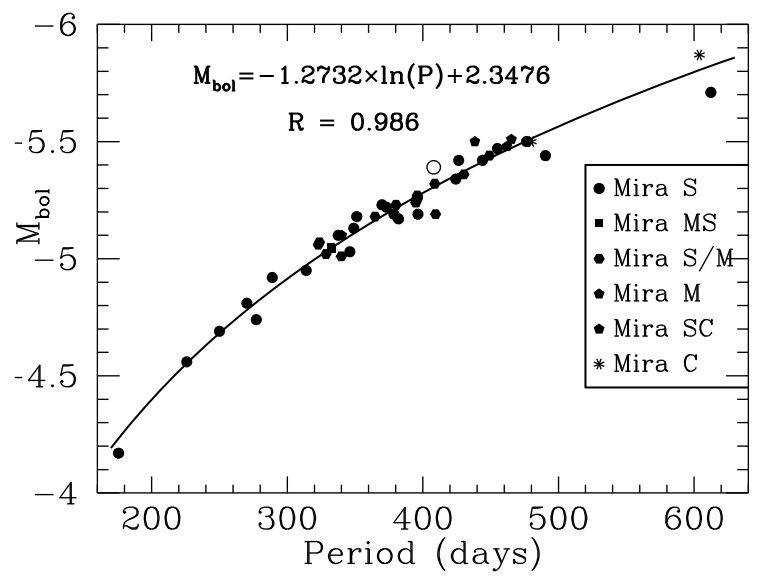

Fig. A.1. The average period-luminosity relation deduced for Miras (filled symbols). The open circle refers to $\chi$ Cyg, whose magnitude, deduced independently from its IR fluxes, its bolometric correction and its Hipparcos distance, fits well in the general relation. The curve represents the written fitting relation, and $R$ is its regression coefficient. See text for further explanations.

distance. The two distance values were then averaged to get a final choice. This choice for the stellar distance was then applied to our apparent bolometric magnitudes, thus deducing our best estimate for the absolute bolometric Magnitude.

Although certainly intricate, the above procedure should minimize the systematic errors of each individual method. The absolute magnitudes thus deduced are plotted as a function of the period in Fig. A.1, thus providing our choice for the average $P-L$ relation, descending primarily from the quoted works, but also from our averaging technique and from our estimate of the bolometric corrections. The derived period-magnitude relation can be expressed by a fitting spline with a high accuracy, as shown on the same graph. In the plot, we also included, as a comparison, a point (the open circle) corresponding to the Mira star $\chi C y g$. For it we did not apply any $P-L$ relation: the absolute magnitude was obtained from directly scaling our apparent bolometric magnitude with the known Hipparcos distance (van Leeuwen 2007a). Although it's only a single point, its good accord with the trend defined by the other sources gives us a consistency check for the technique.

\section{References}

Alcock, C., Axelrod, T. S., Bennett, D. P., et al. 1992, Robotic telescopes in the 1990s, 103rd Annual Meeting of the ASP, Univ. of Wyoming, Laramie, 1991, 193

Allende Prieto, C., Lambert, D. L., \& Asplund, M. 2002, ApJ, 573, L137

Anders, E., \& Grevesse, N. 1989, Geochem. Cosmochem. Acta, 53, 197

Andersen, A. C., Höfner, S., \& Gautschy-Loidl, R. 2003, A\&A, 400, 981

Bedding, T. R., \& Zijlstra, A. A. 1998, ApJ, 506, L47

Bessell, M. S., Castelli, F., \& Plez, B. 1998, A\&A, 333, 231

Bonačić Marinović, A., Izzard, R. G., Lugaro, M., \& Pols, O. R. 2007, A\&A, 469, 1013

Busso, M., Gallino, R., Lambert, D. L., Raiteri, C. M., \& Smith, V. V. 1992, ApJ, 399,218

Busso, M., Lambert, D. L., Beglio, L., et al. 1995, ApJ, 446, 775

Busso, M., Origlia, L., Marengo, M., et al. 1996, A\&A, 311, 253

Busso, M., Gallino, R., \& Wasserburg, G. J. 1999, ARA\&A, 37, 239

Busso, M., Gallino, R., Lambert, D. L., Travaglio, C., \& Smith, V. V. 2001, ApJ, 557,802

Busso, M., Gallino, R., \& Wasserburg, G. J. 2003, PASA, 20, 356

Busso, M., Guandalini, R., Persi, P., Corcione, L., \& Ferrari-Toniolo, M. 2007, AJ, 133, 2310 (Paper II)
Carciofi, A. C., Bjorkman, J. E., \& Magalhães, A. M. 2004, ApJ, 604, 238

Cioni, M.-R. L., Blommaert, J. A. D. L., Groenewegen, M. A. T., et al. 2003, A\&A, 406, 51

Cohen, J. G., Persson, S. E., Elias, J. H., \& Frogel, J. A. 1981, ApJ, 249, 481

Cohen, M., Wheaton, Wm. A., \& Megeath, S. T. 2003, AJ, 126, 1090

Cutri, R. M., Skrutskie, M. F., van Dyk, S., et al. 2003, VizieR On-line Data Catalog: II/246, University of Massachusetts and Infrared Processing and Analysis Center (IPAC/California Institute of Technology)

Feast, M. 1999, IAUS, 191, 109

Feast, M. 2004, IAU Colloq., 193, Proceedings of the conference held 6-11 July, 2003 at Christchurch, New Zealand, ed. D. W. Kurtz, \& K. R. Pollard (San Francisco: ASP), ASP Conf. Proc., 310, 304

Feast, M. W., Glass, I. S., Whitelock, P. A., \& Catchpole, R. M. 1989, MNRAS, 241,375

Glass, I. S. 1999, The Handbook of Infrared Astronomy (Cambridge, England: Cambridge University Press)

Groenewegen, M. A. T., Sevenster, M., Spoon, H. W. W., \& Pérez, I. 2002, A\&A, 390,511

Guandalini, R., Busso, M., Ciprini, S., Silvestro, G., \& Persi, P. 2006, A\&A, 445, 1069 (Paper I)

Habing, H. J. 1996, A\&ARv, 7, 97

Hughes, S. M. G., \& Wood, P. R. 1990, AJ, 99, 784

Izzard, R. G., Jeffery, C. S., \& Lattanzio, J. C. 2007, A\&A, 470, 661

Kerschbaum, F., \& Hron, J. 1996, A\&A, 308, 489

Knapp, G. R., \& Morris, M. 1985, ApJ, 292, 640

Le Bertre, T., Matsuura, M., Winters, J. M., et al. 2001, A\&A, 376, 997

Le Bertre, T., Tanaka, M., Yamamura, I., \& Murakami, H. 2003, A\&A, 403, 943

Marigo, P., Girardi, L., \& Chiosi, C. 2003, A\&A, 403, 225

Merrill, S. P. W. 1952, ApJ, 116, 21

Olivier, E. A., \& Wood, P. R. 2003, ApJ, 584, 1035

Olofsson, A. O. H., Olofsson, G., Hjalmarson, Å., et al. 2003, A\&A, 402, 47

Omont, A., Gilmore, G. F., Alard, C., et al. 2003, A\&A, 403, 975

Salpeter, E. E. 1974, ApJ, 193, 585

Samus, N. N., Durlevich, O. V., et al. 2004, VizieR On-line Data Catalog: II/250, Institute of Astronomy of Russian Academy of Science and Sternberg, State Astronomical Institute of the Moscow State University

Sandin, C., \& Höfner, S. 2003a, A\&A, 398, 253

Sandin, C., \& Höfner, S. 2003b, A\&A, 404, 789

Sedlmayr, E. 1994, Lecture Notes in Physics, 428, 163

Smith, V. V., \& Lambert, D. L. 1986, ApJ, 311, 843

Smith, V. V., \& Lambert, D. L. 1990, ApJS, 72, 387

Stephenson, C. B. 1984, A General Catalogue of Galactic S-Stars, edn.2 (Warner \& Swasey Observatory)

Stephenson, C. B. 1990, AJ, 100, 569

Tuchman, Y. 1999, IAUS, 191, 123

Uttenthaler, S., Hron, J., Lebzelter, T., et al. 2007, A\&A, 463, 251

Van Eck, S., Jorissen, A., Udry, S., et al. 2000, A\&AS, 145, 51

van Leeuwen, F. 2007a, Hipparcos: The new reduction of the raw data (Berlin: Springer)

van Leeuwen, F. 2007b, A\&A, 474, 653

Wachter, A., Schröder, K.-P., Winters, J. M., Arndt, T. U., \& Sedlmayr, E. 2002, A\&A, 384, 452

Whitelock, P., \& Feast, M. 2000, MNRAS, 319, 759

Whitelock, P., Menzies, J., Feast, M., et al. 1994, MNRAS, 267, 711

Whitelock, P. A., Feast, M. W., Marang, F., \& Groenewegen, M. A. T. 2006, MNRAS, 369, 751

Whitelock, P. A., Feast, M. W., \& van Leeuwen, F. 2008, MNRAS, Online Early, 393

Winters J. M., Le Bertre, T., Jeong, K. S., Nyman, L.-Å., \& Epchtein, N. 2003, A\&A, 409, 715

Wood, P. R. 2003, Mass-losing pulsating stars and their circumstellar matter, Workshop, May 13-16, 2002, Sendai, Japan, ed. Y. Nakada, M. Honma, \& M. Seki (Dordrecht: Kluwer Academic Publishers), Astrophys. Space Sci. Library, 283, 3

Wood, P. R., \& Cohen, M. 2001, Post-AGB Objects as a Phase of Stellar Evolution, Proceedings of the Torun Workshop held July 5-7, 2000, ed. R. Szczerba, \& S. K. Górny (Kluwer Academic Publishers), Astrophys. Space Sci. Library, 265, 71

Wood, P. R., \& Sebo, K. M. 1996, MNRAS, 282, 958

Wood, P. R., Alcock, C., Allsman, R. A., et al. 1999, IAUS, 191, 151

Wood, P. R., Olivier, E. A., \& Kawaler, S. D. 2004, ApJ, 604, 800

Yang, X., Chen, P., Wang, J., \& He, J. 2006, AJ, 132, 1468

Zijlstra, A. A., Bedding, T. R., Markwick, A. J., et al. 2004, MNRAS, 352, 325

Zinner, E. 2000, Meteoritics \& Planetary Science, 35, Supplement, A177 
R. Guandalini and M. Busso: The evolutionary status of mass-losing AGB stars. II., Online Material p 1

Table 1. Sample $A$ - first part. Spectral type is from the GCVS catalogue whenever possible, otherwise it is obtained from the SIMBAD Astronomical Database.

\begin{tabular}{cccccc}
\hline \hline $\begin{array}{c}\text { IRAS } \\
\text { name }\end{array}$ & $\begin{array}{c}\text { Other } \\
\text { name }\end{array}$ & $\begin{array}{c}\text { Stephenson } \\
\text { name }\end{array}$ & $\begin{array}{c}\text { Coordinates } \\
\text { ICRS }\end{array}$ & Spectral type & $\begin{array}{c}\text { Var. type } \\
\text { (GCVS) }\end{array}$ \\
\hline $01159+7220$ & S Cas & CSS 28 & $011941.97+723639.3$ & S3,4e-S5,8e & M \\
$19126-0708$ & W Aql & CSS 1115 & $191523.44-070249.9$ & S3,9e-S6,9e & M \\
$19354+5005$ & R Cyg & CSS 1150 & $193649.381+501159.46$ & S2.5,9e-S6,9e(Tc) & M \\
$19486+3247$ & chi Cyg & CSS 1165 & $195033.9220+325450.610$ & S6,2e-S10,4e/MSe & M \\
$23595-1457$ & W Cet & CSS 1346 & $000207.3891-144033.065$ & S6,3e-S9,2e & M \\
$22196-4612$ & pi1 Gru & CSS 1294 & $222244.2053-455652.598$ & S5,7e & SRB \\
$20026+3640$ & AA Cyg & CSS 1188 & $200427.6055+364900.465$ & S7,5-S7.5,6(MpTc) & SRB \\
$20120-4433$ & RZ Sgr & CSS 1196 & $201528.4049-442437.480$ & S4,4ep & SRB \\
$03452+5301$ & WX Cam & CSS 82 & $034903.77+531059.2$ & S5,8 & LB \\
\hline $23070+0824$ & GZ Peg & CSS 1322 & $230931.4570+084037.778$ & M4SIII & SRA \\
$15492+4837$ & ST Her & CSS 903 & $155046.6248+482858.856$ & M6-7IIIaS & SRB \\
$00192-2020$ & T Cet & CSS 8 & $002146.2737-200328.885$ & M5-6SIIe & SRC \\
$05374+3153$ & NO Aur & CSS 149 & $054042.0504+315514.187$ & M2SIab & LC \\
$22476+4047$ & RX Lac & CSS 1308 & $224956.8992+410304.312$ & M7.5Se & SRB \\
\hline $00213+3817$ & R And & CSS 9 & $002401.9469+383437.328$ & S3,5e-S8,8e/M7e & M \\
$22521+1640$ & HR Peg & CSS 1315 & $225435.6272+165630.601$ & S5,1/M4 & SRB \\
$17553+4521$ & OP Her & - & $175648.5274+452103.063$ & M5IIb-IIIa/S & SRB \\
\hline $13372-7136$ & LY Mus & CSS 826 & $134113.5883-715205.767$ & M4III & LB \\
$18058-3658$ & - & CSS 1023 & $180917.1853-365757.614$ & M2II-III & - \\
\hline $19111+2555$ & S Lyr & CSS 1112 & $191311.79+260028.3$ & SCe & M \\
\hline $15194-5115$ & II Lup & CSS 886 & $152304.91-512559.0$ & C & M \\
\hline
\end{tabular}

Table 2. Sample $A$ - second part.

\begin{tabular}{|c|c|c|c|c|c|c|c|c|c|c|c|}
\hline $\begin{array}{l}\text { Source } \\
\text { name }\end{array}$ & $\begin{array}{c}J \\
{[\mathrm{Jy}]}\end{array}$ & $\begin{array}{c}H \\
{[\mathrm{Jy}]}\end{array}$ & $\begin{array}{c}K \\
{[\mathrm{Jy}]}\end{array}$ & $\begin{array}{c}8.8] \\
{[\mathrm{Jy}]}\end{array}$ & $\begin{array}{l}{[9.8]} \\
{[\mathrm{Jy}]}\end{array}$ & $\begin{array}{c}{[11.7]} \\
{[\mathrm{Jy}]}\end{array}$ & $\begin{array}{c}12.5] \\
{[\mathrm{Jy}]}\end{array}$ & $\begin{array}{c}D \\
{[\mathrm{Jy}]}\end{array}$ & $\begin{array}{c}E \\
{[\mathrm{Jy}]}\end{array}$ & Mid-IR Data Origin & $\begin{array}{c}\text { ISO } \\
\text { TDT number }\end{array}$ \\
\hline S Cas & 56.6 & 168 & 244 & 232 & 316 & 304 & 281 & 235 & 185 & ISO-SWS1 & 41602133 \\
\hline W Aql & 388 & 822 & 1113 & 969 & 1099 & 1043 & 789 & 625 & 488 & ISO-SWS1 & 16402335 \\
\hline R Cyg & 200 & 288 & 302 & 77.3 & 87.8 & 79.3 & 69.2 & 52.8 & 29.7 & ISO-SWS1 & 42201625 \\
\hline chi Cyg & 1365 & 2823 & 3176 & 1408 & 1655 & 1579 & 1095 & 736 & 487 & ISO-SWS1 & 15900437 \\
\hline W Cet & 74.1 & 113 & 101 & 12.7 & 12.4 & 10.5 & 10.9 & 8.3 & 4.5 & ISO-SWS1 & 37802225 \\
\hline pil Gru & 3080 & - & 5812 & 544 & 632 & 679 & 683 & 505 & 365 & ISO-SWS1 & 34402039 \\
\hline AA Cyg & 238 & 389 & 375 & 42.6 & 42.5 & 42.9 & 36.8 & 27.5 & 16.9 & ISO-SWS1 & 36401817 \\
\hline RZ Sgr & 139 & 206 & 190 & 25.0 & 26.2 & 25.7 & 24.5 & 21.8 & 21.0 & ISO-SWS1 & 14100818 \\
\hline WX Cam & 43.5 & 79.3 & 87.6 & 8.1 & 7.9 & 8.4 & 16.6 & 9.3 & 4.0 & ISO-SWS1 & 81002721 \\
\hline GZ Peg & 735 & 1100 & 965 & 88.6 & 78.9 & 62.4 & 56.8 & 40.9 & 21.0 & ISO-SWS1 & 37600306 \\
\hline ST Her & 804 & 1162 & 1098 & 166 & 186 & 200 & 187 & 149 & 104 & ISO-SWS1 & 41901305 \\
\hline $\mathrm{T}$ Cet & 1009 & 1596 & 1403 & 172 & 163 & 172 & 171 & 134 & 83.0 & ISO-SWS1 & $55502308-37801819$ \\
\hline NO Aur & 226 & 362 & 273 & 33.2 & 41.6 & 46.3 & 36.1 & 23.3 & 17.2 & ISO-SWS1 & 86603434 \\
\hline RX Lac & 465 & 737 & 681 & 91.0 & 85.4 & 81.9 & 68.6 & 50.3 & 28.8 & ISO-SWS1 & 78200427 \\
\hline R And & 247 & 506 & 596 & 193 & 264 & 248 & 210 & 176 & 135 & ISO-SWS1 & 40201723 \\
\hline HR Peg & 191 & 327 & 256 & 27.0 & 24.4 & 20.9 & 20.0 & 12.6 & 7.8 & ISO-SWS1 & 37401910 \\
\hline OP Her & 416 & 731 & 556 & 63.5 & 58.1 & 46.4 & 37.8 & 30.9 & 16.0 & ISO-SWS1 & 77800625 \\
\hline$\overline{\text { LY Mus }}$ & 221 & 334 & 300 & 28.4 & 24.7 & 19.7 & 16.4 & 12.9 & 7.6 & ISO-SWS1 & 13201304 \\
\hline CSS 1023 & 33.2 & 51.7 & 39.9 & 2.3 & 2.2 & 1.5 & 1.4 & 0.83 & 0.46 & ISO-SWS1 & 14100603 \\
\hline S Lyr & 8.2 & 13.2 & 17.9 & 20.0 & 23.2 & 25.5 & 24.4 & 22.1 & 15.7 & ISO-SWS1 & 52000546 \\
\hline II Lup & 3.7 & 25.5 & 99.0 & 860 & 852 & 860 & 681 & 506 & 391 & ISO-SWS6 & 29700401 \\
\hline
\end{tabular}


R. Guandalini and M. Busso: The evolutionary status of mass-losing AGB stars. II., Online Material p 2

Table 3. Sample $A$ - third part. The indication I. - E. (Intrinsic - Extrinsic) is given according to the suggestions from Van Eck et al. (2000) and Yang et al. (2006). In discordant cases we prefer the choice by Van Eck et al. (2000) and show the one of Yang et al. (2006) in parenthesis. For few sources, for which neither study offers a suggestion, we infer that they are extrinsic from their low intrinsic luminosity: in the tables they are underlined.

\begin{tabular}{|c|c|c|c|c|c|c|c|}
\hline $\begin{array}{l}\text { Source } \\
\text { name }\end{array}$ & $\begin{array}{l}\text { Var. type } \\
\text { (GCVS) }\end{array}$ & $\begin{array}{c}\text { Period } \\
\text { (GCVS) }\end{array}$ & $\begin{array}{l}\text { Distance } \\
(\mathrm{kpc})\end{array}$ & $\begin{array}{l}\text { Min. - Max. } \\
(\mathrm{kpc})\end{array}$ & $\begin{array}{c}\text { Ref. } \\
\text { distance }\end{array}$ & $\begin{array}{l}\text { Bol. magnitudes } \\
\text { ISO integration }\end{array}$ & I. $-\mathrm{E}$. \\
\hline S Cas & $\mathrm{M}$ & 612.43 & 0.85 & - & $P-L /$ this paper & -5.71 & I \\
\hline W Aql & M & 490.43 & 0.34 & - & $P-L /$ this paper & -5.44 & I \\
\hline R Cyg & $\mathrm{M}$ & 426.45 & 0.55 & - & $P-L /$ this paper & -5.42 & - \\
\hline chi Cyg & $\mathrm{M}$ & 408.05 & 0.18 & $0.15-0.22$ & Hip. / van Leeuwen (2007a) & -5.39 & I \\
\hline W Cet & M & 351.31 & 0.83 & - & $P-L /$ this paper & -5.18 & I \\
\hline pi1 Gru & SRB & 150 & 0.16 & $0.15-0.19$ & Hip. / van Leeuwen (2007a) & -5.75 & I \\
\hline AA Cyg & SRB & 212.7 & - & - & - & - & I \\
\hline RZ Sgr & SRB & 223.2 & - & - & - & - & I \\
\hline WX Cam & LB & - & - & - & - & - & I \\
\hline GZ Peg & SRA & 92.66 & 0.24 & $0.22-0.26$ & Hip. / van Leeuwen (2007a) & -5.02 & $\bar{E}$ \\
\hline ST Her & SRB & 148 & 0.30 & $0.25-0.36$ & Hip. / van Leeuwen (2007a) & -5.64 & I \\
\hline T Cet & SRC & 158.9 & 0.27 & $0.24-0.31$ & Hip. / van Leeuwen (2007a) & -5.63 & I \\
\hline NO Aur & LC & - & 0.60 & $0.47-0.83$ & Hip. / van Leeuwen (2007a) & -5.73 & I \\
\hline RX Lac & SRB & 650 & - & - & - & - & I \\
\hline R And & $\bar{M}$ & 409.33 & 0.41 & - & $P-L /$ this paper & -5.19 & I \\
\hline HR Peg & SRB & 50 & 0.41 & $0.36-0.50$ & Hip. / van Leeuwen (2007a) & -4.75 & I \\
\hline OP Her & SRB & 120.5 & 0.30 & $0.27-0.32$ & Hip. / van Leeuwen (2007a) & -4.92 & - \\
\hline LY Mus & LB & - & 0.29 & $0.26-0.33$ & Hip. / van Leeuwen (2007a) & -4.12 & $\mathrm{E}$ \\
\hline CSS 1023 & - & - & - & - & - & - & $\mathrm{E}$ \\
\hline S Lyr & $\mathrm{M}$ & 438.4 & 2.27 & - & $P-L /$ this paper & -5.50 & $\mathrm{I}$ \\
\hline II Lup & $\mathrm{M}$ & - & 0.59 & - & Groenewegen et al. (2002) & -4.82 & - \\
\hline
\end{tabular}

Table 4. Sample $B$ - first part. Suggestions from Smith \& Lambert (1986) for Spectral Type: 1 RS Cnc: M6eIIIaS. 2 V1743 Cyg: M5IIIaS. 3 V1981 Cyg: M4IIIaS.

\begin{tabular}{cccccc}
\hline \hline $\begin{array}{c}\text { IRAS } \\
\text { name }\end{array}$ & $\begin{array}{c}\text { Other } \\
\text { name }\end{array}$ & $\begin{array}{c}\text { Stephenson } \\
\text { name }\end{array}$ & $\begin{array}{c}\text { Coordinates } \\
\text { ICRS }\end{array}$ & Spectral type & $\begin{array}{c}\text { Var. type } \\
\text { (GCVS) }\end{array}$ \\
\hline $05199-0842$ & V1261 Ori & CSS 133 & $052218.6453-083958.034$ & S & Algol type \\
\hline $04497+1410$ & omi Ori & CSS 114 & $045231.9621+141502.311$ & M3.2IIIaS & SRB \\
$10226+0902$ & DE Leo & - & $102515.1951+084705.441$ & M2IIIabS & SRB \\
$07245+4605$ & Y Lyn & CSS 347 & $072811.6109+455926.207$ & M6SIb-II & SRC \\
$07392+1419$ & NZ Gem & CSS 382 & $074203.2185+141230.612$ & M3II-IIIS & SR \\
\hline $06457+0535$ & V613 Mon & CSS 260 & $064822.2963+053230.050$ & M2/S5,1 & SRB \\
$09076+3110$ & RS Cnc & CSS 589 & $091038.7990+305747.300$ & M6eIb-II/S & SRC \\
$03377+6303$ & BD Cam & CSS 79 & $034209.3250+631300.501$ & S5,3/M4III & LB \\
$07095+6853$ & AA Cam & CSS 312 & $071452.0703+684815.380$ & M5/S & LB \\
$13079-8931$ & BQ Oct & CSS 804 & $143529.5001-894618.182$ & M4III/S5,1 & LB \\
\hline $12272-4127$ & V928 Cen & CSS 796 & $122957.8871-414409.242$ & M2II-III & SRB \\
$19323+4909$ & V1743 Cyg & - & $193341.6068+491544.347$ & M4.5III & SRB \\
- & V1981 Cyg & - & $210224.1993+444727.528$ & M4s... & SRB \\
$08214-3807$ & V436 Pup & CSS 500 & $082316.9344-381709.884$ & M1III & LB \\
- & V2141 Cyg & CSS 1254 & $205753.1771+444717.336$ & M1 & LB \\
$12106-3350$ & V335 Hya & - & $121312.9423-340730.981$ & M4III & LB \\
$14510-6052$ & CR Cir & CSS 867 & $145456.9389-610433.027$ & M2/M3II & LC \\
$16418-1359$ & - & CSS 937 & $164442.1936-140448.553$ & M1III & - \\
\hline $13136-4426$ & UY Cen & CSS 816 & $131631.8300-444215.741$ & SC & SR \\
\hline $16425-1902$ & - & CSS 938 & $164530.1769-190812.939$ & K5II & - \\
$20076+3331$ & - & CSS 1194 & $200932.9873+334053.851$ & K5III & - \\
\hline
\end{tabular}


R. Guandalini and M. Busso: The evolutionary status of mass-losing AGB stars. II., Online Material p 3

Table 5. Sample $B$ - second part.

\begin{tabular}{ccccccccccc}
\hline \hline $\begin{array}{c}\text { Source } \\
\text { name }\end{array}$ & $\begin{array}{c}J \\
{[\mathrm{Jy}]}\end{array}$ & $\begin{array}{c}H \mathrm{Jy}] \\
{[\mathrm{Jy}]}\end{array}$ & $\begin{array}{c}{[8.8]} \\
{[\mathrm{Jy}]}\end{array}$ & $\begin{array}{c}{[9.8]} \\
{[\mathrm{Jy}]}\end{array}$ & $\begin{array}{c}{[11.7]} \\
{[\mathrm{Jy}]}\end{array}$ & $\begin{array}{c}{[12.5]} \\
{[\mathrm{Jy}]}\end{array}$ & $\begin{array}{c}D \\
{[\mathrm{Jy}]}\end{array}$ & $\begin{array}{c}E \\
{[\mathrm{Jy}]}\end{array}$ & Mid-IR data origin \\
\hline V1261 Ori & 73.8 & 111 & 93.1 & 16.4 & 16.5 & 19.6 & 21.3 & - & - & IRAS-LRS \\
\hline Omi Ori & 1022 & 1587 & 1227 & 103 & 82.4 & 64.8 & 60.9 & - & - & IRAS-LRS \\
DE Leo & 178 & 234 & 184 & 34.0 & 30.7 & 34.4 & 36.4 & - & - & IRAS-LRS \\
Y Lyn & 876 & 1448 & 1256 & 132 & 150 & 121 & 107 & - & - & IRAS-LRS \\
NZ Gem & 369 & 566 & 399 & 38.9 & 34.5 & 32.6 & 32.5 & - & - & IRAS-LRS \\
\hline V613 Mon & 49.6 & 74.3 & 68.9 & 5.2 & - & - & 3.3 & 2.0 & - & MSX \\
RS Cnc & 3065 & 4324 & 3742 & 512 & 693 & 493 & 436 & - & - & TIRCAM2 \\
BD Cam & 439 & 630 & 521 & 62.3 & 52.5 & 43.6 & 41.2 & - & - & IRAS-LRS \\
AA Cam & 146 & 220 & 185 & - & - & - & - & - & - & - \\
BQ Oct & 139 & 196 & 170 & 24.8 & 22.5 & 23.5 & 23.6 & - & - & IRAS-LRS \\
\hline V928 Cen & 196 & 267 & 225 & 25.4 & 21.9 & 20.3 & 20.5 & - & - & IRAS-LRS \\
V1743 Cyg & 259 & 421 & 335 & 40.4 & 36.4 & 34.5 & 34.2 & - & - & IRAS-LRS \\
V1981 Cyg & 197 & 279 & 245 & 28.9 & - & - & 16.3 & 10.8 & 4.7 & MSX \\
V436 Pup & 139 & 198 & 165 & 17.1 & - & - & 10.3 & 7.0 & 2.7 & MSX \\
V2141 Cyg & 147 & 216 & 195 & 20.8 & - & - & 12.2 & 8.2 & 3.5 & MSX \\
V335 Hya & 611 & 946 & 833 & 88.6 & 78.7 & 67.4 & 63.3 & - & - & IRAS-LRS \\
CR Cir & 75.1 & 117 & 99.6 & 9.9 & - & - & 5.8 & 3.8 & - & MSX \\
CSS 937 & 104 & 169 & 151 & 15.7 & 15.9 & 20.1 & 21.6 & - & - & IRAS-LRS \\
\hline UY Cen & 184 & 360 & 350 & 66.7 & 62.9 & 61.4 & 55.6 & - & - & IRAS-LRS \\
\hline CSS 938 & 142 & 200 & 175 & 20.4 & 19.7 & 21.4 & 23.2 & - & - & IRAS-LRS \\
CSS 1194 & 31.1 & 41.5 & 37.0 & 2.9 & - & - & 2.4 & 0.96 & - & MSX \\
\hline
\end{tabular}

Table 6. Sample $B$ - third part.

\begin{tabular}{|c|c|c|c|c|c|c|c|}
\hline $\begin{array}{c}\text { Source } \\
\text { name }\end{array}$ & $\begin{array}{l}\text { Var. type } \\
\text { (GCVS) }\end{array}$ & $\begin{array}{c}\text { Period } \\
(\text { GCVS })\end{array}$ & $\begin{array}{c}\text { Distance } \\
(\mathrm{kpc})\end{array}$ & $\begin{array}{l}\text { Min. - Max. } \\
(\mathrm{kpc})\end{array}$ & $\begin{array}{c}\text { Ref. } \\
\text { distance }\end{array}$ & $\begin{array}{l}\text { Bol. magnitudes } \\
\text { Bol. corrections }\end{array}$ & I. $-\mathrm{E}$. \\
\hline V1261 Ori & Algol Type & - & 0.29 & $0.23-0.38$ & Hip. / van Leeuwen (2007a) & -2.71 & $\mathrm{E}$ \\
\hline omi Ori & SRB & 30 & 0.20 & $0.17-0.23$ & Hip. / van Leeuwen (2007a) & -4.89 & I \\
\hline DE Leo & SRB & - & 0.31 & $0.27-0.37$ & Hip. / van Leeuwen (2007a) & -3.60 & $E$ \\
\hline Y Lyn & SRC & 110 & 0.25 & $0.20-0.33$ & Hip. / van Leeuwen (2007a) & -5.33 & $\overline{\mathrm{I}}$ \\
\hline NZ Gem & SR & - & 0.39 & $0.32-0.51$ & Hip. / van Leeuwen (2007a) & -5.06 & E \\
\hline V613 Mon & SRB & - & 0.50 & $0.35-0.84$ & Hip. / van Leeuwen (2007a) & -3.76 & $\bar{E}$ \\
\hline $\mathrm{RS} \mathrm{Cnc}$ & SRC & 120 & 0.14 & $0.13-0.15$ & Hip. / van Leeuwen (2007a) & -5.21 & I \\
\hline BD Cam & LB & - & 0.16 & $0.15-0.17$ & Hip. / van Leeuwen (2007a) & -3.40 & $\mathrm{E}$ \\
\hline AA Cam & LB & - & 0.78 & $0.50-1.82$ & Hip. / van Leeuwen (2007a) & - & I \\
\hline BQ Oct & LB & - & 0.49 & $0.41-0.60$ & Hip. / van Leeuwen (2007a) & -4.55 & I \\
\hline V928 Cen & SRB & - & 0.23 & $0.21-0.25$ & Hip. / van Leeuwen (2007a) & -3.27 & $\mathrm{E}$ \\
\hline V1743 Cyg & SRB & 40 & 0.41 & $0.38-0.44$ & Hip. / van Leeuwen (2007a) & -4.94 & - \\
\hline V1981 Cyg & SRB & - & 0.30 & $0.27-0.33$ & Hip. / van Leeuwen (2007a) & -3.96 & $E$ \\
\hline V436 Pup & LB & - & 0.33 & $0.30-0.38$ & Hip. / van Leeuwen (2007a) & -3.75 & $\bar{E}$ \\
\hline V2141 Cyg & LB & - & 0.38 & $0.31-0.51$ & Hip. / van Leeuwen (2007a) & -4.24 & $\underline{E}$ \\
\hline V335 Hya & LB & - & 0.36 & $0.31-0.43$ & Hip. / van Leeuwen (2007a) & -5.68 & - \\
\hline CR Cir & $\mathrm{LC}$ & - & 0.31 & $0.25-0.42$ & Hip. / van Leeuwen (2007a) & -3.08 & $\mathrm{E}$ \\
\hline CSS 937 & - & - & 0.42 & $0.31-0.66$ & Hip. / van Leeuwen (2007a) & -4.11 & E \\
\hline UY Cen & SR & 114.6 & 0.69 & $0.47-1.33$ & Hip. / van Leeuwen (2007a) & -6.05 & $\mathrm{I}$ \\
\hline CSS 938 & - & - & 0.25 & $0.20-0.33$ & Hip. / van Leeuwen (2007a) & -3.15 & $\bar{E}$ \\
\hline CSS 1194 & - & - & 0.36 & $0.30-0.45$ & Hip. / van Leeuwen (2007a) & -2.34 & $\underline{E}$ \\
\hline
\end{tabular}


Table 7. Sample $C$ - first part.

\begin{tabular}{|c|c|c|c|c|c|}
\hline $\begin{array}{l}\text { IRAS } \\
\text { name }\end{array}$ & $\begin{array}{l}\text { Other } \\
\text { name }\end{array}$ & $\begin{array}{c}\text { Stephenson } \\
\text { name }\end{array}$ & $\begin{array}{c}\text { Coordinates } \\
\text { ICRS }\end{array}$ & Spectral type & $\begin{array}{l}\text { Var. type } \\
\text { (GCVS) }\end{array}$ \\
\hline $04352+6602$ & T Cam & CSS 103 & $044008.8768+660848.654$ & $\mathrm{~S} 4,7 \mathrm{e}-\mathrm{S} 8.5,8 \mathrm{e}$ & $\bar{M}$ \\
\hline $06571+5524$ & R Lyn & CSS 283 & $070118.0093+551949.766$ & $\mathrm{~S} 2.5,5 \mathrm{e}-\mathrm{S} 6,8 \mathrm{e}:$ & $\mathrm{M}$ \\
\hline $07043+2246$ & R Gem & CSS 307 & $070721.2744+224212.736$ & $\mathrm{~S} 2,9 \mathrm{e}-\mathrm{S} 8,9 \mathrm{e}(\mathrm{Tc})$ & $\mathrm{M}$ \\
\hline $07092+0735$ & WX CMi & CSS 316 & $071157.45+072959.3$ & $\mathrm{Se}$ & $\mathrm{M}$ \\
\hline $12417+6121$ & S UMa & CSS 803 & $124356.676+610535.51$ & S0,9e-S5,9e & $\mathrm{M}$ \\
\hline $15030-4116$ & GI Lup & CSS 872 & $150616.31-412814.1$ & $\mathrm{~S} 7,8 \mathrm{e}$ & $\mathrm{M}$ \\
\hline $23554+5612$ & WY Cas & CSS 1345 & $235801.30+562913.5$ & S6,5pe & $\mathrm{M}$ \\
\hline $00135+4644$ & $X$ And & CSS 6 & $001609.57+470044.8$ & $\mathrm{~S} 2,9 \mathrm{e}-\mathrm{S} 5,5 \mathrm{e}$ & $\mathrm{M}$ \\
\hline $00435+4758$ & U Cas & CSS 12 & $004621.371+481438.72$ & $\mathrm{~S} 3,5 \mathrm{e}-\mathrm{S} 8,6 \mathrm{e}$ & $\mathrm{M}$ \\
\hline $06062+2830$ & GH Aur & CSS 191 & $060927.71+282943.4$ & $\mathrm{~S}$ & $\mathrm{M}$ \\
\hline 07197-1451 & TT CMa & CSS 341 & $072202.00-145656.7$ & $\mathrm{~S}$ & M \\
\hline 07584-2051 & EX Pup & CSS 443 & $080038.31-205935.3$ & $\mathrm{~S} 2,4 \mathrm{e}$ & M \\
\hline 11179-6135 & RY Car & CSS 742 & $112011.39-615216.8$ & $\mathrm{~S} 7,8 \mathrm{e}$ & M \\
\hline $13226-6302$ & NZ Cen & CSS 820 & $132602.52 \quad-63 \quad 1828.5$ & $\mathrm{Se}$ & $\mathrm{M}$ \\
\hline $14212+8403$ & R Cam & CSS 856 & $141751.0439+834953.861$ & $\mathrm{~S} 2,8 \mathrm{e}-\mathrm{S} 8,7 \mathrm{e}$ & $\mathrm{M}$ \\
\hline $17478-2957$ & V762 Sgr & CSS 1001 & $175104.04-295830.9$ & S6,4 & $\mathrm{M}$ \\
\hline $17490-3502$ & V407 Sco & CSS 1004 & $175225.53-350317.5$ & $\mathrm{Se}$ & $\mathrm{M}$ \\
\hline $19166+0318$ & ER Aql & CSS 1121 & $191906.99+032405.2$ & $\mathrm{~S}$ & M \\
\hline $23376+6304$ & V441 Cas & CSS 1338 & $233958.92+632055.1$ & $\mathrm{~S}$ & $\mathrm{M}$ \\
\hline $23489+6235$ & EO Cas & CSS 1342 & $235127.30+625147.0$ & $\mathrm{Se}$ & $\mathrm{M}$ \\
\hline $00001+4826$ & IW Cas & CSS 1347 & $000244.22+484250.9$ & $\mathrm{~S} 4.5,9 \mathrm{e}$ & $\mathrm{M}$ \\
\hline- & - & CSS2 10 & $025133.00+575034.5$ & $\mathrm{~S}$ & M \\
\hline $10237-6135$ & AU Car & CSS 679 & $102529.74-615059.1$ & MS & $\mathrm{M}$ \\
\hline $10349-6203$ & RX Car & CSS 690 & $103645.82-62 \quad 1916.8$ & MS & M \\
\hline $02143+4404$ & W And & CSS 49 & $021732.9606+441817.766$ & S6,1e-S9,2e/M4-M1 & $\bar{M}$ \\
\hline 07149+0111 & RR Mon & CSS 326 & $071731.54+010541.5$ & $\mathrm{~S} 7,2 \mathrm{e}-\mathrm{S} 8,2 \mathrm{e} / \mathrm{M} 6-10$ & $\mathrm{M}$ \\
\hline $07545-4400$ & SU Pup & CSS 436 & $075612.0813-440833.254$ & $\mathrm{M} / \mathrm{S} 4,2 \mathrm{e}$ & $\mathrm{M}$ \\
\hline 09338-5349 & UU Vel & CSS 614 & $093533.21-540325.9$ & $\mathrm{M} 2 \mathrm{e} / \mathrm{S} 7,8 \mathrm{e}$ & $\mathrm{M}$ \\
\hline $17001-3651$ & RT Sco & CSS 954 & $170332.56-365513.7$ & S7,2/M6e-M7e & M \\
\hline $20213+0047$ & V865 Aql & CSS 1211 & $202354.6422+005644.794$ & M6-M7/S7,5e: & $\mathrm{M}$ \\
\hline $00445+3224$ & RW And & CSS 14 & $004718.92+324108.6$ & $\mathrm{M} 5 \mathrm{e}-\mathrm{M} 10 \mathrm{e} / \mathrm{S} 6,2 \mathrm{e}$ & M \\
\hline $07103-0258$ & AK Mon & CSS 319 & $071249.91-030329.0$ & M5/S5,1 & M \\
\hline $17521-2907$ & V745 Sgr & CSS 1007 & $175519.00-290754.4$ & $\mathrm{Se} / \mathrm{M}$ & M \\
\hline $20044+5750$ & S Cyg & CSS 1191 & $200529.85+575909.1$ & $\mathrm{~S} 2.5,1 \mathrm{e} / \mathrm{M} 3.5-\mathrm{M} 7 \mathrm{e}$ & $\mathrm{M}$ \\
\hline $20369+3742$ & FF Cyg & CSS 1232 & $203851.71+375323.2$ & $\mathrm{~S} 6,8 \mathrm{e} / \mathrm{M} 4 \mathrm{e}$ & M \\
\hline $03499+4730$ & FG Per & CSS 85 & $035330.2+473904$ & M9 & $\bar{M}$ \\
\hline $13163-6031$ & TT Cen & CSS 817 & $131935.016-604646.26$ & $\mathrm{CSe}$ & $\bar{M}$ \\
\hline $18586-1249$ & ST Sgr & CSS 1096 & $190129.20-124534.0$ & $\mathrm{C} 4,3 \mathrm{e}-\mathrm{S} 9,5 \mathrm{e}$ & M \\
\hline $21540+4806$ & LX Cyg & CSS 1286 & $215557.03+482052.6$ & SC3e-S5,5e: & M \\
\hline $18575-0139$ & VX Aql & CSS 1093 & $190009.61-013456.8$ & C9,1p/M0ep & $\mathrm{M}$ \\
\hline $01097+6154$ & V418 Cas & CSS 23 & $011259.89+621047.6$ & - & $\mathrm{M}$ \\
\hline
\end{tabular}


R. Guandalini and M. Busso: The evolutionary status of mass-losing AGB stars. II., Online Material p 5

Table 8. Sample $C$ - second part.

\begin{tabular}{|c|c|c|c|c|c|c|c|c|c|c|}
\hline $\begin{array}{c}\text { Source } \\
\text { name }\end{array}$ & $\begin{array}{c}J \\
{[\mathrm{Jy}]}\end{array}$ & $\begin{array}{c}H \\
{[\mathrm{Jy}]}\end{array}$ & $\begin{array}{c}K \\
{[\mathrm{Jy}]}\end{array}$ & $\begin{array}{c}{[8.8]} \\
{[\mathrm{Jy}]}\end{array}$ & $\begin{array}{c}{[9.8]} \\
{[\mathrm{Jy}]}\end{array}$ & $\begin{array}{c}{[11.7]} \\
{[\mathrm{Jy}]}\end{array}$ & $\begin{array}{c}{[12.5]} \\
{[\mathrm{Jy}]}\end{array}$ & $\begin{array}{c}D \\
{[\mathrm{Jy}]}\end{array}$ & $\begin{array}{c}E \\
\text { [Jy] }\end{array}$ & Mid-IR data origin \\
\hline T Cam & 152 & 306 & 315 & 62.1 & 53.4 & 48.8 & 47.1 & - & - & IRAS-LRS \\
\hline R Lyn & 51.7 & 77.7 & 91.1 & 29.9 & 29.9 & 29.2 & 28.5 & - & - & IRAS-LRS \\
\hline R Gem & 155 & 226 & 174 & 24.1 & - & - & 21.4 & 16.3 & 7.5 & MSX \\
\hline WX CMi & 8.9 & 13.8 & 17.3 & 8.3 & - & - & 9.2 & 6.3 & 4.1 & MSX \\
\hline S UMa & 26.3 & 43.4 & 41.4 & 4.5 & - & 2.8 & 3.1 & - & - & TIRCAM2 \\
\hline GI Lup & 61.7 & 115 & 128 & - & - & - & - & - & - & - \\
\hline WY Cas & 64.5 & 94.4 & 120 & 48.8 & 55.6 & 58.2 & 55.0 & - & - & IRAS-LRS \\
\hline$X$ And & 18.1 & 37.3 & 43.3 & 23.6 & 23.6 & 24.9 & 26.6 & - & - & IRAS-LRS \\
\hline U Cas & 29.6 & 45.0 & 47.1 & 15.2 & 15.6 & 19.4 & 20.3 & - & - & IRAS-LRS \\
\hline GH Aur & 4.4 & 7.8 & 10.2 & 1.5 & - & - & 1.8 & - & - & MSX \\
\hline TT CMa & 29.6 & 50.1 & 54.3 & 12.3 & - & - & 14.1 & 10.8 & 7.7 & MSX \\
\hline EX Pup & 3.9 & 5.9 & 5.8 & 0.54 & - & - & - & - & - & MSX \\
\hline RY Car & 8.3 & 18.2 & 25.1 & 11.0 & - & - & 8.9 & 5.6 & 3.2 & MSX \\
\hline NZ Cen & 8.3 & 15.7 & 17.2 & 9.1 & - & - & 9.3 & 6.3 & 5.2 & MSX \\
\hline R Cam & 47.2 & 70.8 & 69.5 & 7.1 & - & - & - & 2.8 & - & MSX \\
\hline V762 Sgr & 15.0 & 35.9 & 44.5 & 13.5 & - & - & 14.4 & 9.3 & 5.1 & MSX \\
\hline V407 Sco & 9.8 & 18.1 & 19.4 & 6.1 & - & - & 5.6 & 3.8 & - & MSX \\
\hline ER Aql & 34.4 & 61.5 & 62.3 & 10.1 & - & - & 6.9 & 4.7 & - & MSX \\
\hline V441 Cas & 5.9 & 11.6 & 14.3 & 2.0 & - & - & 1.5 & - & - & MSX \\
\hline EO Cas & 11.8 & 21.4 & 30.9 & 9.3 & - & - & 7.5 & 4.8 & 3.0 & MSX \\
\hline IW Cas & 23.3 & 47.4 & 50.9 & 52.9 & 58.5 & 61.8 & 60.5 & - & - & IRAS-LRS \\
\hline CSS2 10 & 1.3 & 2.8 & 3.1 & 0.36 & - & - & - & - & - & MSX \\
\hline AU Car & 5.9 & 9.0 & 10.3 & 2.1 & - & - & - & - & - & MSX \\
\hline RX Car & 5.0 & 7.7 & 8.9 & 2.1 & - & - & 1.7 & - & - & MSX \\
\hline W And & 368 & 643 & 591 & 185 & 198 & 163 & 143 & - & - & IRAS-LRS \\
\hline RR Mon & 24.1 & 42.5 & 52.8 & 21.3 & - & - & 18.0 & 12.7 & 7.2 & MSX \\
\hline SU Pup & 28.5 & 43.8 & 45.5 & 22.3 & 26.6 & 29.1 & 29.3 & - & - & IRAS-LRS \\
\hline UU Vel & 23.5 & 46.9 & 51.6 & 13.6 & - & - & 11.5 & 7.5 & 4.1 & MSX \\
\hline RT Sco & 285 & 460 & 512 & 162 & - & - & 163 & 108 & 67.4 & MSX \\
\hline V865 Aql & 126 & 191 & 199 & 36.5 & 33.8 & 35.2 & 35.8 & - & - & IRAS-LRS \\
\hline RW And & 95.9 & 132 & 131 & 48.8 & 52.8 & 53.0 & 50.1 & - & - & IRAS-LRS \\
\hline AK Mon & 9.3 & 13.1 & 14.7 & 3.1 & - & - & 2.8 & 2.2 & - & MSX \\
\hline V745 Sgr & 38.2 & 75.8 & 82.5 & 18.5 & - & - & 16.1 & 11.3 & 7.4 & MSX \\
\hline S Cyg & 11.8 & 14.0 & 16.5 & 2.0 & - & - & 1.5 & 0.74 & - & MSX \\
\hline FF Cyg & 37.8 & 65.8 & 82.0 & 8.5 & - & - & 6.4 & 4.9 & - & MSX \\
\hline FG Per & 3.2 & 6.0 & 6.8 & 1.1 & - & - & - & - & - & MSX \\
\hline TT Cen & 19.5 & 48.9 & 54.7 & 15.5 & - & - & 16.3 & 10.1 & 8.0 & MSX \\
\hline ST Sgr & 97.7 & 148 & 149 & 61.6 & 63.2 & 61.1 & 56.4 & - & - & IRAS-LRS \\
\hline LX Cyg & 14.2 & 28.0 & 44.9 & 10.2 & - & - & 9.4 & 5.1 & - & MSX \\
\hline VX Aql & 13.5 & 33.6 & 37.7 & 9.3 & - & - & 10.6 & 6.8 & 3.5 & MSX \\
\hline V418 Cas & 11.0 & 16.0 & 22.8 & 12.5 & - & - & 12.2 & 7.9 & 5.5 & MSX \\
\hline
\end{tabular}


R. Guandalini and M. Busso: The evolutionary status of mass-losing AGB stars. II., Online Material p 6

Table 9. Sample $C$ - third part.

\begin{tabular}{|c|c|c|c|c|c|c|}
\hline $\begin{array}{c}\text { Source } \\
\text { name }\end{array}$ & $\begin{array}{l}\text { Var. type } \\
\text { (GCVS) }\end{array}$ & $\begin{array}{l}\text { Period } \\
\text { (GCVS) }\end{array}$ & $\begin{array}{l}\text { Distance } \\
(\mathrm{kpc})\end{array}$ & $\begin{array}{c}\text { Ref. } \\
\text { distance }\end{array}$ & $\begin{array}{c}\text { Bol. magnitudes } \\
P-L \text { method }\end{array}$ & $\overline{\text { I. }-\mathrm{E} \text {. }}$ \\
\hline T Cam & $\mathrm{M}$ & 373.2 & 0.50 & $P-L /$ this paper & -5.22 & I \\
\hline R Lyn & M & 378.75 & 0.95 & $P-L /$ this paper & -5.19 & I \\
\hline R Gem & M & 369.91 & 0.66 & $P-L /$ this paper & -5.23 & I \\
\hline WX CMi & M & 420.1 & 2.35 & $P-L /$ this paper & -5.31 & - \\
\hline S UMa & M & 225.87 & 0.96 & $P-L /$ this paper & -4.56 & I \\
\hline GI Lup & M & 326.2 & 0.80 & $P-L /$ this paper & - & I \\
\hline WY Cas & M & 476.56 & 0.97 & $P-L /$ this paper & -5.50 & I \\
\hline$X$ And & M & 346.18 & 1.31 & $P-L /$ this paper & -5.03 & I \\
\hline U Cas & M & 277.19 & 1.08 & $P-L /$ this paper & -4.74 & I \\
\hline GH Aur & M & 349 & 2.64 & $P-L /$ this paper & -5.13 & I \\
\hline TT CMa & M & 314 & 1.08 & $P-L /$ this paper & -4.95 & I \\
\hline EX Pup & M & 289 & 3.02 & $P-L /$ this paper & -4.92 & - \\
\hline RY Car & M & 424.3 & 1.95 & $P-L /$ this paper & -5.34 & I \\
\hline NZ Cen & M & 382 & 2.22 & $P-L /$ this paper & -5.17 & I \\
\hline R Cam & M & 270.22 & 0.84 & $P-L /$ this paper & -4.81 & $\mathrm{E}$ \\
\hline V762 Sgr & M & 444 & 1.50 & $P-L /$ this paper & -5.42 & I \\
\hline V407 Sco & M & 396 & 2.11 & $P-L /$ this paper & -5.26 & I \\
\hline ER Aql & M & 337.6 & 1.04 & $P-L /$ this paper & -5.10 & I \\
\hline V441 Cas & M & 175.6 & 1.40 & $P-L /$ this paper & -4.17 & $\underline{E}$ \\
\hline EO Cas & M & 455 & 1.83 & $P-L /$ this paper & -5.47 & $\bar{I}$ \\
\hline IW Cas & M & 396.38 & 1.34 & $P-L /$ this paper & -5.19 & - \\
\hline CSS2 10 & M & 250 & 3.78 & $P-L /$ this paper & -4.69 & - \\
\hline AU Car & $\mathrm{M}$ & 332 & 2.54 & $P-L /$ this paper & -5.05 & - \\
\hline RX Car & M & 332.8 & 2.75 & $P-L /$ this paper & -5.04 & - \\
\hline W And & $\mathrm{M}$ & 395.93 & 0.38 & $P-L /$ this paper & -5.27 & $\mathrm{I}$ \\
\hline RR Mon & M & 394.7 & 1.28 & $P-L /$ this paper & -5.24 & I \\
\hline SU Pup & M & 339.8 & 1.26 & $P-L /$ this paper & -5.01 & I \\
\hline UU Vel & M & 408.9 & 1.31 & $P-L /$ this paper & -5.32 & I \\
\hline RT Sco & M & 449.04 & 0.45 & $P-L /$ this paper & -5.44 & I \\
\hline V865 Aql & M & 364.8 & 0.62 & $P-L /$ this paper & -5.18 & - \\
\hline RW And & M & 430.3 & 0.86 & $P-L /$ this paper & -5.36 & - \\
\hline AK Mon & M & 328.6 & 2.12 & $P-L /$ this paper & -5.02 & - \\
\hline V745 Sgr & $\mathrm{M}$ & 380.2 & 0.99 & $P-L /$ this paper & -5.23 & - \\
\hline S Cyg & M & 322.93 & 1.94 & $P-L /$ this paper & -5.06 & I \\
\hline FF Cyg & M & 323.82 & 0.87 & $P-L /$ this paper & -5.07 & I \\
\hline FG Per & $\mathrm{M}$ & 340.3 & 3.16 & $P-L /$ this paper & -5.10 & - \\
\hline TT Cen & $\bar{M}$ & 462 & 1.39 & $P-L /$ this paper & -5.48 & - \\
\hline ST Sgr & M & 395.12 & 0.76 & $P-L /$ this paper & -5.24 & I \\
\hline LX Cyg & $\mathrm{M}$ & 465.3 & 1.53 & $P-L /$ this paper & -5.51 & I \\
\hline VX Aql & $\overline{\mathrm{M}}$ & $\begin{array}{c}604 \\
\text { Zijlstra et al. (2004) }\end{array}$ & 1.99 & $P-L /$ this paper & -5.87 & $\bar{I}$ \\
\hline V418 Cas & $\bar{M}$ & 480 & 2.24 & $P-L /$ this paper & -5.50 & - \\
\hline
\end{tabular}


R. Guandalini and M. Busso: The evolutionary status of mass-losing AGB stars. II., Online Material p 7

Table 10. Sample $D$ - distance estimates.

\begin{tabular}{cccc}
\hline \hline $\begin{array}{c}\text { Source } \\
\text { name }\end{array}$ & $\begin{array}{c}\text { Apparent bol. } \\
\text { magnitudes }\end{array}$ & $\begin{array}{c}\text { Distance } \\
(\mathrm{kpc})\end{array}$ & $\begin{array}{c}\text { Min. - Max. distance } \\
(\mathrm{kpc})\end{array}$ \\
\hline NX Per & 7.61 & 3.57 & $2.97-4.30$ \\
NU Pup & 8.58 & 5.56 & $4.63-6.69$ \\
DK CMa & 6.06 & 1.75 & $1.45-2.10$ \\
EW Pup & 7.04 & 2.74 & $2.28-3.29$ \\
WY Pyx & 5.01 & 1.08 & $0.90-1.29$ \\
V2434 Oph & 4.95 & 1.05 & $0.87-1.26$ \\
V342 Ser & 6.55 & 2.18 & $1.82-2.63$ \\
V471 Sct & 5.85 & 1.59 & $1.32-1.91$ \\
V427 Sct & 6.88 & 2.54 & $2.11-3.06$ \\
V1959 Cyg & 6.47 & 2.11 & $1.76-2.54$ \\
\hline PR Nor & 4.51 & 0.85 & $0.71-1.03$ \\
PZ Vul & 8.43 & 5.20 & $4.33-6.25$ \\
GY Lac & 7.31 & 3.11 & $2.58-3.74$ \\
V928 Cas & 8.22 & 4.73 & $3.93-5.69$ \\
V508 Aur & 8.28 & 4.86 & $4.04-5.84$ \\
V1992 Cyg & 7.90 & 4.07 & $3.38-4.89$ \\
V1850 Cyg & 8.56 & 5.53 & $4.60-6.65$ \\
V1242 Cyg & 8.12 & 4.50 & $3.74-5.41$ \\
\hline
\end{tabular}

